\title{
Atomic Force Microscopy on Biological Materials Related to Pathological Conditions
}

\author{
Andreas Stylianou $\mathbb{D}^{1}{ }^{1}$ Stylianos-Vasileios Kontomaris, ${ }^{2,3}$ Colin Grant, ${ }^{4}$ \\ and Eleni Alexandratou ${ }^{5}$ \\ ${ }^{1}$ Cancer Biophysics Laboratory, Department of Mechanical and Manufacturing Engineering, University of Cyprus, \\ Nicosia 2238, Cyprus \\ ${ }^{2}$ Mobile Radio Communications Laboratory, School of Electrical and Computer Engineering, National Technical University \\ of Athens, Iroon Polytechniou, Athens 15780, Greece \\ ${ }^{3}$ Athens Metropolitan College, Sorou 74, Marousi 15125, Greece \\ ${ }^{4}$ Hitachi High-Technologies Europe, Techspace One, Keckwick Lane, Warrington WA4 4AB, UK \\ ${ }^{5}$ Biomedical Optics and Applied Biophysics Laboratory, School of Electrical and Computer Engineering, National Technical \\ University of Athens, Iroon Polytechniou, Athens 15780, Greece
}

Correspondence should be addressed to Andreas Stylianou; stylianou.c.andreas.1@ucy.ac.cy

Received 7 December 2018; Revised 23 February 2019; Accepted 7 March 2019; Published 12 May 2019

Academic Editor: Daniele Passeri

Copyright (c) 2019 Andreas Stylianou et al. This is an open access article distributed under the Creative Commons Attribution License, which permits unrestricted use, distribution, and reproduction in any medium, provided the original work is properly cited.

\begin{abstract}
Atomic force microscopy (AFM) is an easy-to-use, powerful, high-resolution microscope that allows the user to image any surface and under any aqueous condition. AFM has been used in the investigation of the structural and mechanical properties of a wide range of biological matters including biomolecules, biomaterials, cells, and tissues. It provides the capacity to acquire high-resolution images of biosamples at the nanoscale and allows at readily carrying out mechanical characterization. The capacity of AFM to image and interact with surfaces, under physiologically relevant conditions, is of great importance for realistic and accurate medical and pharmaceutical applications. The aim of this paper is to review recent trends of the use of AFM on biological materials related to health and sickness. First, we present AFM components and its different imaging modes and we continue with combined imaging and coupled AFM systems. Then, we discuss the use of AFM to nanocharacterize collagen, the major fibrous protein of the human body, which has been correlated with many pathological conditions. In the next section, AFM nanolevel surface characterization as a tool to detect possible pathological conditions such as osteoarthritis and cancer is presented. Finally, we demonstrate the use of AFM for studying other pathological conditions, such as Alzheimer's disease and human immunodeficiency virus (HIV), through the investigation of amyloid fibrils and viruses, respectively. Consequently, AFM stands out as the ideal research instrument for exploring the detection of pathological conditions even at very early stages, making it very attractive in the area of bio- and nanomedicine.
\end{abstract}

\section{Introduction}

Atomic force microscopy (AFM) belongs to the scanning probe microscopy (SPM) family and was developed following on from the scanning tunnelling microscopy (STM), which was awarded the 1986 Nobel Prize in Physics. AFM is a SPM that records interactions between a sharp probe (the AFM tip) at the end of a small cantilever and the sample surface. Since its invention in the 1980s, it has become a fundamental technique in the fields of surface science. AFM has several advantages over the other microscopic techniques, such as scanning and transmission electron microscopy (SEM and TEM) and optical microscopy (including fluorescent and confocal laser scanning microscopy). First of all, AFM provides quantifiable and accurate surface height information, down to the Angstrom level-while other microscopes can give topographical contrast, they cannot provide three-dimensional topographies. Measurements 
and images captured by AFM can be made in air, aqueous, or vacuum conditions at a range of temperatures. Plus, the sample preparations are considerably easier than those used for TEM. After image acquisition, the AFM user can perform mechanical/electrical/magnetic property characterization of sample surface, offering a combination of qualitative and quantitative information [1]. AFM is characterized as a nondestructive tool that can operate under different conditions (air and liquid) since it requires only the basic sample preparation (e.g., does not require dehydration, labeling with fluorescent dyes or antibodies, or surface coating) [2-5].

AFM was developed in 1986 by Binnig and colleagues [6] and commercial AFMs began to appear in the early 1990s [7]. Since its invention, it has rapidly become a popular method for high-resolution nanoscale imaging and mechanical property characterization of a broad range of samples, especially biological materials $[3,8]$. The key requirement for AFM imaging is the probe, a sharp tip mounted on a cantilever (Figure 1). A huge range of tip shapes and geometries are commercially available along with a range of cantilever spring constants $(0.001$ to $2000 \mathrm{~N} / \mathrm{m})$ and cantilever coatings that can allow imaging of delicate soft matter without causing damage or even make indentations in glass.

1.1. Force versus Distance Curves. In this section, the applied forces during the interaction between the AFM tip and the sample's surface will be presented. These forces are attractive or repulsive depending on the distance between the AFM tip and the sample (Figure 2(a)). More specifically, if the abovementioned distance is big enough, the resultant force is attractive (van der Waals force) [9]. On the contrary, for small distances, the resultant force is repulsive due to the overlapping of electron orbitals between the tip and sample [9]. The aforementioned forces can be approximated using the Lennard-Jones potential (Figure 2(b)) [10]:

$$
V(d)=4 V_{\mathrm{m}}\left[\left(\frac{d_{\mathrm{m}}}{d}\right)^{12}-\left(\frac{d_{\mathrm{m}}}{d}\right)^{6}\right]
$$

where $V(d)$ and $d$ are the intermolecular potential and the distance between the two atoms or molecules, respectively; $V_{\mathrm{m}}$ is the well depth (a measure of how much the two particles attract each other); and $d_{\mathrm{m}}$ is the distance at which $V=0$.

As it is presented in Figure 2(a), when the distance between the AFM tip and the sample's surface is equal to $d_{\text {eq }}$ (where $d_{\text {eq }}$ is the equilibrium distance), the resultant force on the tip is zero. In addition, in the case that $d>d_{\text {eq }}$, the resultant force is attractive (negative net force, $F_{\mathrm{T}}=F_{\mathrm{R}}-$ $F_{\mathrm{A}}<0$, where $F_{\mathrm{R}}$ is the repulsive force, $F_{\mathrm{A}}$ the attractive force, and $F_{\mathrm{T}}$ the total force on the tip). On the contrary, in the case that $d<d_{\mathrm{eq}}$, the resultant force is repulsive (positive net force, $F_{\mathrm{T}}=F_{\mathrm{R}}-F_{\mathrm{A}}>0$ ).

1.2. Applied Forces in the AFM Operation Modes. The categorization of the imaging modes is based on the type of the applied forces on the sample. More specifically, in the contact mode, the tip is always in contact with the sample and the resultant force is repulsive. On the contrary, in the

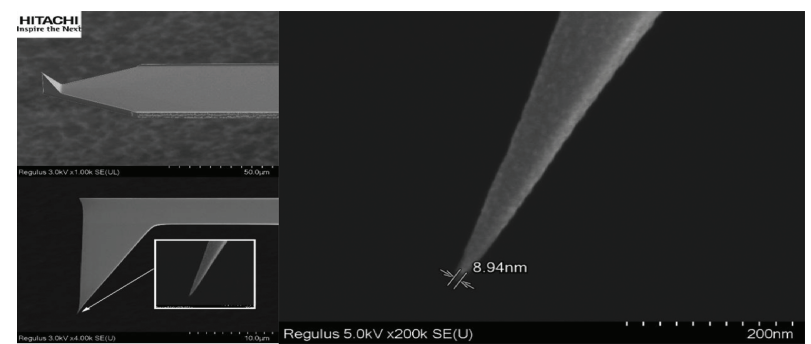

FIgUre 1: AFM tip. SEM images (Hitachi Regulus SU 8230) of an Olympus AC160 AFM probe, with a measured tip diameter of $9 \mathrm{~nm}$ (unpublished data obtained by Colin Grant).

noncontact mode, the tip is never in contact with the sample's surface; thus, the resultant force is attractive. In the tapping mode, the resultant force can be attractive or repulsive since the tip alternately moves toward to or away from the sample's surface. Last but not least, in the force mode (in which the sample's mechanical properties must be tested), the sample is moving toward the tip; hence, the net force is initially attractive and then repulsive. The selection of an AFM imaging mode depends on the characteristics of the sample that must be tested. For example, for a nonbiological sample, the contact mode is preferable due to the fact that there is no possibility to cause permanent damage to the sample. In the case of very soft biological samples, the noncontact mode seems to be the preferred choice. However, it presents severe limitations (e.g., it must be applied in a high vacuum environment) $[11,12]$, and as a result, the tapping mode is mostly used for imaging soft biological samples. In Figure 3, the total force with respect to distance (between the tip and the sample) is presented. The blue box presents the range of interaction forces in the contact region, and the green and grey boxes the range of interaction forces in the noncontact region and the intermittent region (tapping mode), respectively.

The AFM instrument itself consists of a laser beam aligned to the back of a cantilever, which is then reflected onto a position-sensitive detector (Figure 4(a)). The accurate movement of the tip over the surface is achieved with piezoelectric elements in the $x, y, z$ frame. The probe scans over the sample surface where any changes of the laser spot position on the detector are recorded and acted upon by feedback electronics, resulting in the accurate representation of the sample surface (Figure 4(a)).

One of the unique features of AFM is that once the user has found a region of interest or interesting feature, the cantilever tip can be used to apply a user-determined force on the sample. In AFM force spectroscopy, with careful calibration of cantilever spring constant, applied forces can be used to compress the sample, generating a force vs. indentation data. By using the AFM nanoindentation procedure [13-15], the stiffness/elasticity of the specimen can be recorded and Young's modulus maps of the sample's surface can be generated [16-20]. Additionally, further research is ongoing for the improvement of the existing mathematical models that are used for the acquisition of quantitative data from AFM modes [21]. 


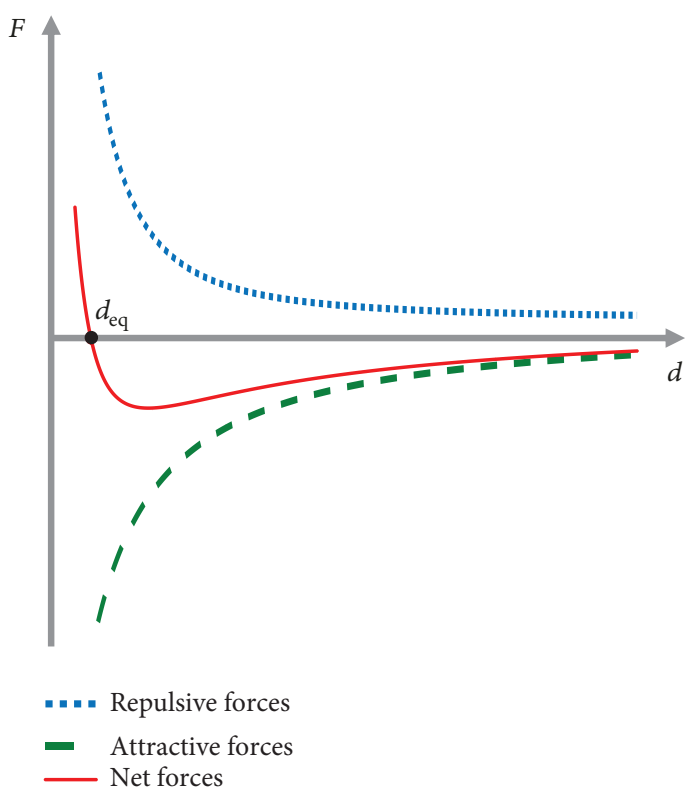

(a)

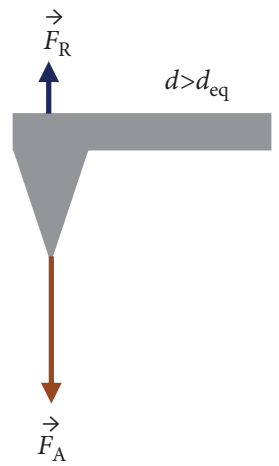

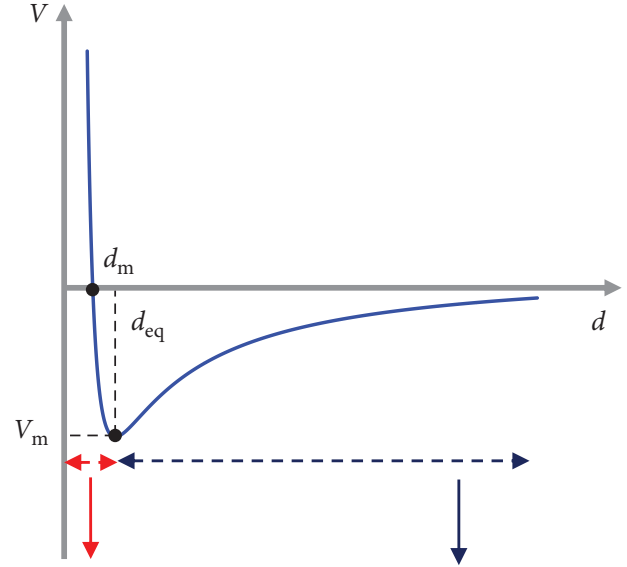

Repulsive resultant force
Attractive resultant force (b)

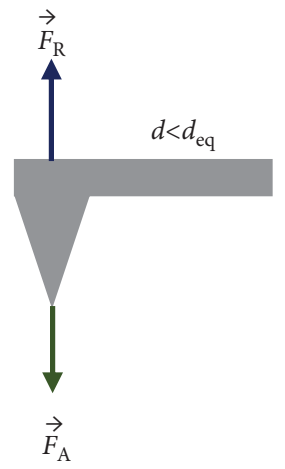

(c)

Figure 2: (a) Force-distance curves. Interaction forces versus distance between the tip and the sample's surface. (b) The Lennard-Jones potential. If $d>d_{\text {eq }}$, the resultant force is attractive, and if $d<d_{\text {eq }}$, the resultant force is repulsive. In the case that $d=d_{\text {eq }}$, the resultant force is zero. (c) Repulsive and attractive forces on the tip. Forces applied on the AFM tip in three different regions.

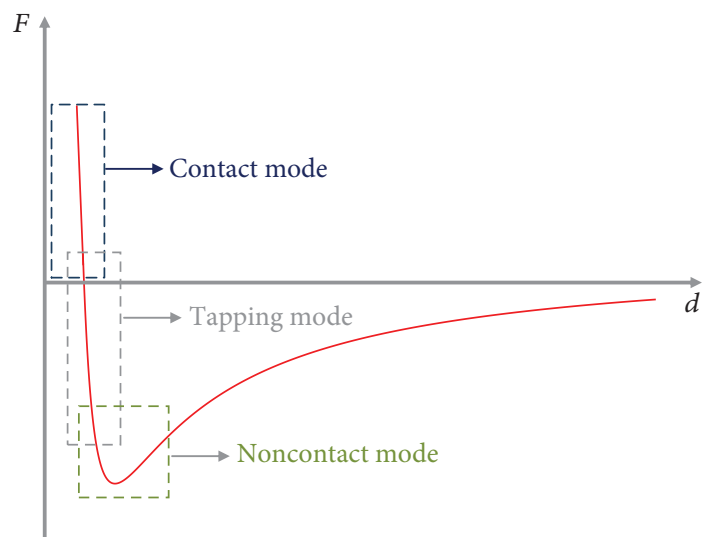

FIGURE 3: Force versus distance: the blue box represents the range of interaction forces in the contact mode (repulsive net force). The grey box and the green box represent the range of the interaction forces in the intermittent and in the noncontact regions, respectively.

\section{Atomic Force Microscopy: Imaging Modes}

Many other microscopes have different kinds of "modes" in order to extract more information about a surface. For example, SEM utilize secondary or backscattered electrons in order to image and provide information on topography and chemical composition, respectively. Optical microscopes can operate in a brightfield, darkfield, polarized, or phase contrast mode, depending on the optics that are used. Similarly, the AFM can operate in a number of different modes (Figures 4(b)-4(g)).

In the tapping mode (also known as intermittent mode, dynamic contact mode, and AC mode), the cantilever is tuned to its resonant frequency at a measured amplitude (Figure 4(b)); any changes in the oscillation amplitude are recorded as the tip scans the surface. Imaging with this dynamic mode is considered very gentle on sample surfaces, capable of high resolution, and almost all lateral forces are eliminated [3, 22]. 


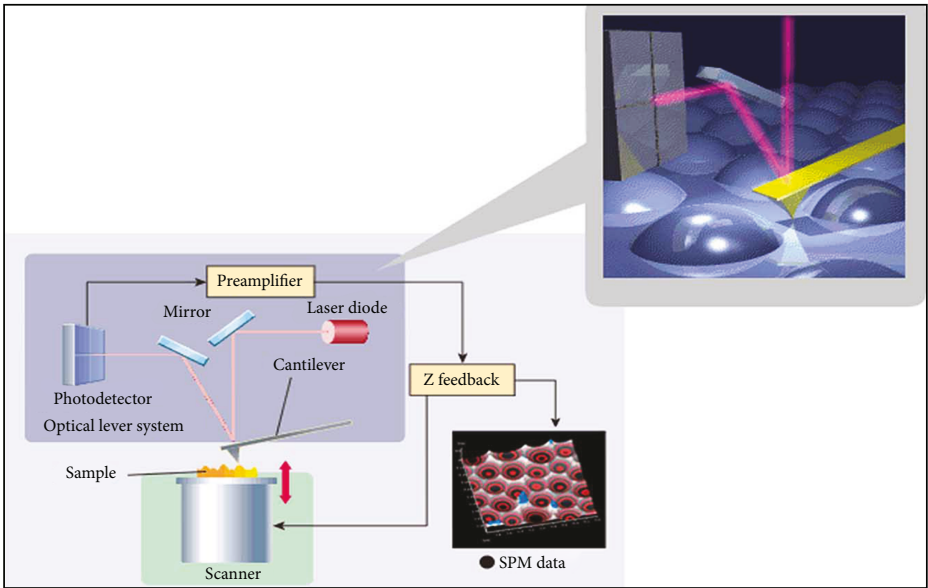

(a)

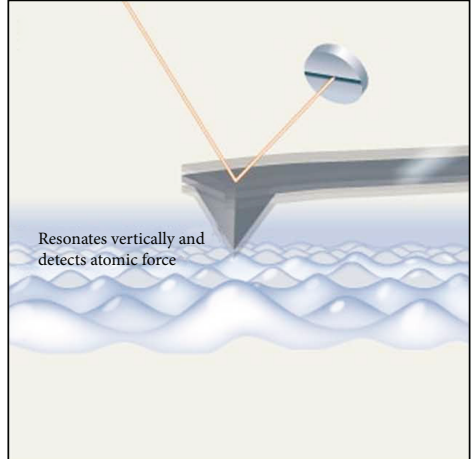

(b)

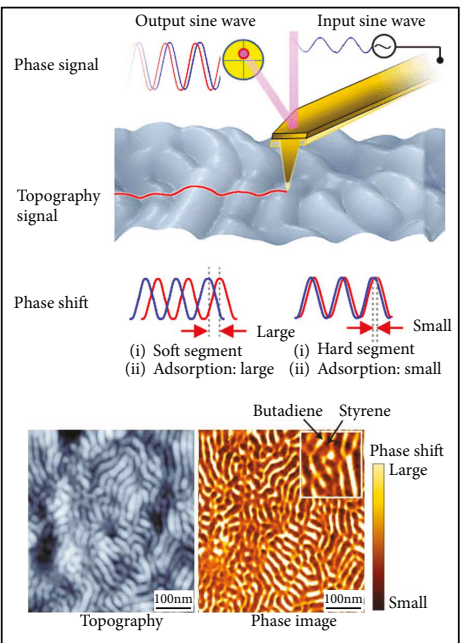

(e)

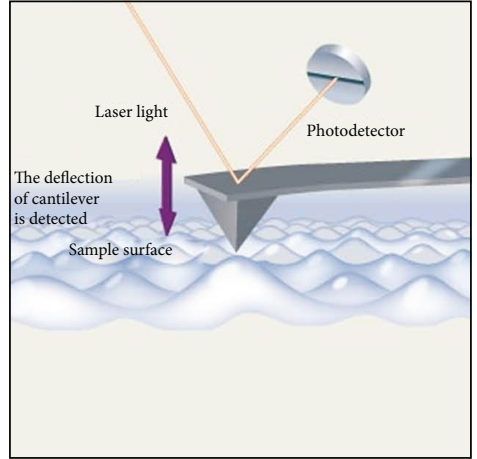

(c)

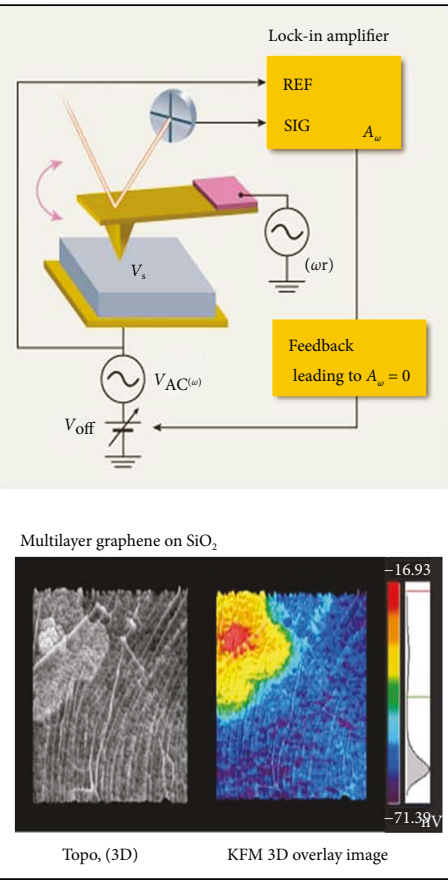

(f)

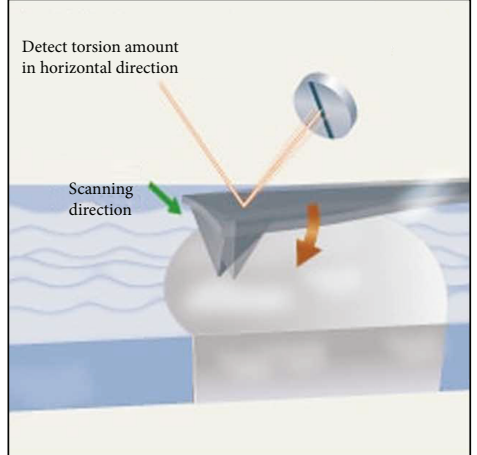

(d)
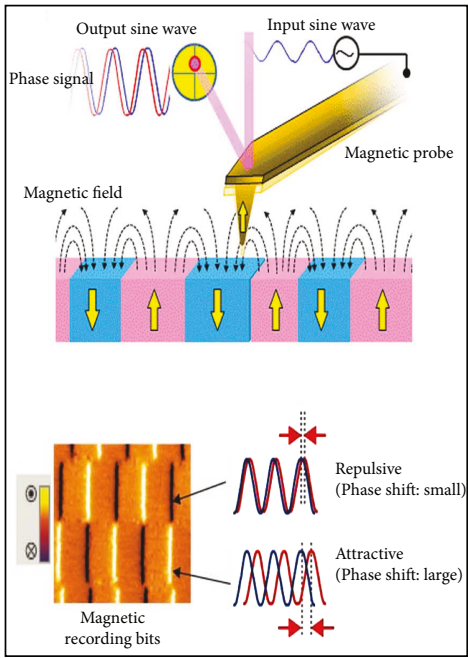

(g)

FIGURE 4: AFM working principles and modes: (a) generalized schematic of AFM of cantilever with the laser reflecting onto the photodetector. (b) Tapping/dynamic mode. (c) Contact mode. (d) Friction/lateral mode. (e) Phase imaging from dynamic mode, where the contrast (butadiene/styrene blend) relates to surface properties. (f) Kelvin probe electrical imaging; contrast shows surface potential (work function) of a graphene flake on $\mathrm{SiO}_{2}$ substrate. (g) Magnetic mode imaging, where a magnetic coated cantilever scans just above the surface and the phase shift relates to attraction/repulsion of magnetic domains (images courtesy of Hitachi High Technology, Tokyo, Japan). 
In the contact mode, the tip is always in contact with the sample surface at a user-determined constant force (Figure 4(c)). Here, the user can track rougher, stiffer surfaces better and faster than the tapping mode. Friction mode imaging (Figure $4(\mathrm{~d})$ ) is a form of static mode (contact mode). This mode is appropriate for measuring the friction of a surface as the side to side twisting of the cantilever by a torque, measured as the probe raster scans along the surface. In the frictional force mode, here, the torsional changes (frictional features) in the photodetector are recorded simultaneously with the vertical changes (topographic features). Most AFM users will use this to gain qualitative frictional contrast of their surfaces; however, with appropriate calibration of the lateral cantilever spring constant [23], a surface frictional coefficient can be calculated.

During the tapping mode, simultaneously with topography images, phase images can be acquired [7]. In phase imaging, the system monitors the phase lag between the signal that drives the cantilever oscillation and its output signal (Figure 4(e)). Phase images can be used for assessing variations in composition, adhesion, and viscoelastic properties of surfaces $[20,24]$.

Other tapping mode-based characterizations include the Kelvin probe (Figure 4(f)) and magnetic mode (Figure 4(g)). Kelvin force microscopy (KFM) is one among a number of electrical characterizations that AFM can carry out. In this technique, a contact potential difference is measured between a conductive AFM probe and the sample of interest. This contact potential difference is negated by the application of a direct external bias of the same magnitude; which is then equal to the difference in work function between the tip and sample [25]. Magnetic imaging mode can image magnetic domain structures of a surface when a magneticcoated cantilever scans the surface [26]. Here, the oscillating probe first scans the surface to get topographical features, but then, the probe elevates off the surface by a small distance and the attraction/repulsion is recorded.

AFM can also operate in a noncontact mode where the AFM tip does not touch at all the sample surface [27], but this mode is not frequently used for biological sample characterization.

In the mechanical mapping mode, the AFM measures the sample stiffness, in terms of Young's modulus values, through the nanoindentation technique. In AFM nanoindentation, the AFM collects indentation-force curves on the sample of interest while these curves are fitted using linearelastic contact mechanical models, such as the Hertz model, in order to estimate Young's modulus [7]. It should be noted that the selection of the contact mechanics models to transform force-indentation curves into elastic moduli is the most fundamental and longstanding issue [28]. Modulus maps can then be formed by indenting in an organized array over a surface [16]. This technique can be very slow, as for example, a $64 \times 64$-pixel modulus map, with one indentation per second would take over an hour. Most AFM manufacturers have developed fast force mapping modes to speed up this procedure where indents are taken at $\mathrm{kHz}$ magnitude, meaning a $512 \times 512$ mechanical map can be taken in minutes. However, at this level of loading rate, the modulus results lack accuracy when indenting viscoelastic time-dependent materials. Further, the tip radius, which is vitally important for the modulus calculation, will be increasing during the imaging procedure.

To avoid this tip blunting effect during nanoindentation mapping, new mechanical imaging techniques have emerged. Contact resonance is a very useful mechanical mapping technique for materials ranging between $50 \mathrm{MPa}$ and $200 \mathrm{GPa}$ and consists of oscillating the samples, while undertaking a contact mode image. To date, this technique has been used only in few biological materials (for instance, for bone material [29]) although this technique does work well in air or under aqueous conditions [30].

In the multifrequency, AFM employs the detection of multiple cantilever frequencies (higher harmonics and/or higher flexural eigenmodes) which provides information concerning the tip-sample nonlinearity [31, 32]. Multiharmonic mode uses alterations in the amplitude, the phase of the oscillator, and other relevant harmonics in order to provide quantitative local property maps $[30,33]$. Multiharmonic, like bimodal AFM which requires the simultaneous excitation of the first two eigenmodes of the cantilever, enables the simultaneous mapping of Young's modulus and the deformation and the topography of the sample [34]. As a result, these modes can be used for investigating complex cellular and biomolecular systems, in health and disease, and offering a detailed quantitative multiparametric characterization [35].

Viscoelastic mapping is another very novel mode that has its roots in research on multifrequency and bimodal AFM. This technique is a dynamic force-based mode that provides both imaging of the topography and maps of nanomechanical properties of soft-matter surfaces [36, 37]. Here, the cantilever is oscillated at two eigenmode frequenciesthe first mode records the surface features and loss tangent data (amplitude modulation AM), while the second mode records frequency variations (frequency modulation FM) which relates to stiffness. AM-FM offers nanomechanical information of the specimen such as Young's modulus and contact stiffness [38] and has several advantages including fast scanning, high spatial resolution, and low forces applied to the sample. Although these modes are quite new, they have been used for investigating several biological samples, including proteins; small biological fibrils, like amyloid fibrils; lipid membranes; and viruses [39-42]. Also, it has been demonstrated that malign and benign cell lines present significant differences in their viscoelastic response [43].

\section{Combined Imaging and Coupled AFM Systems and Their Applications}

3.1. General. Although AFM presents high sensitivity and resolution on imaging and investigating biological samples at the nanoscale, it can lack other important information such as cellular components and biochemical functions [4, 44].

For example, optical microscopic imaging, especially using fluorescence, is another high-performance research tool that can provide complementary information. Fluorescence 


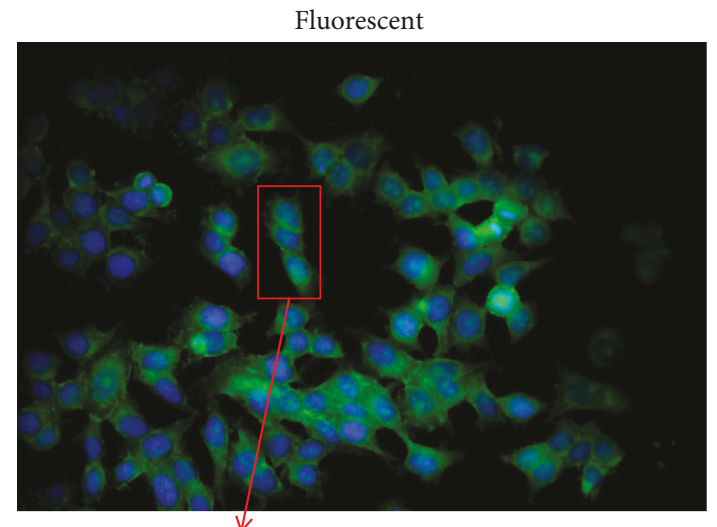

(a)

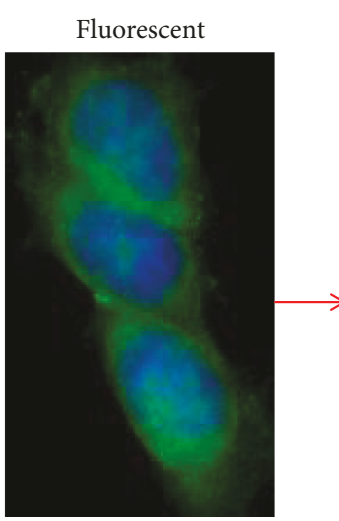

(b)
AFM

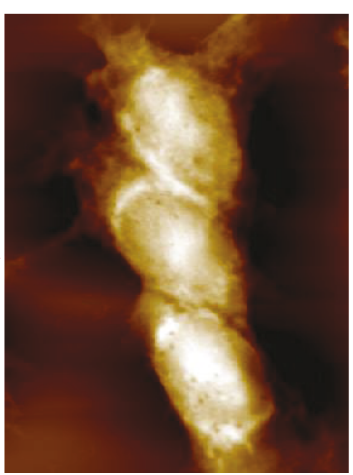

(c)

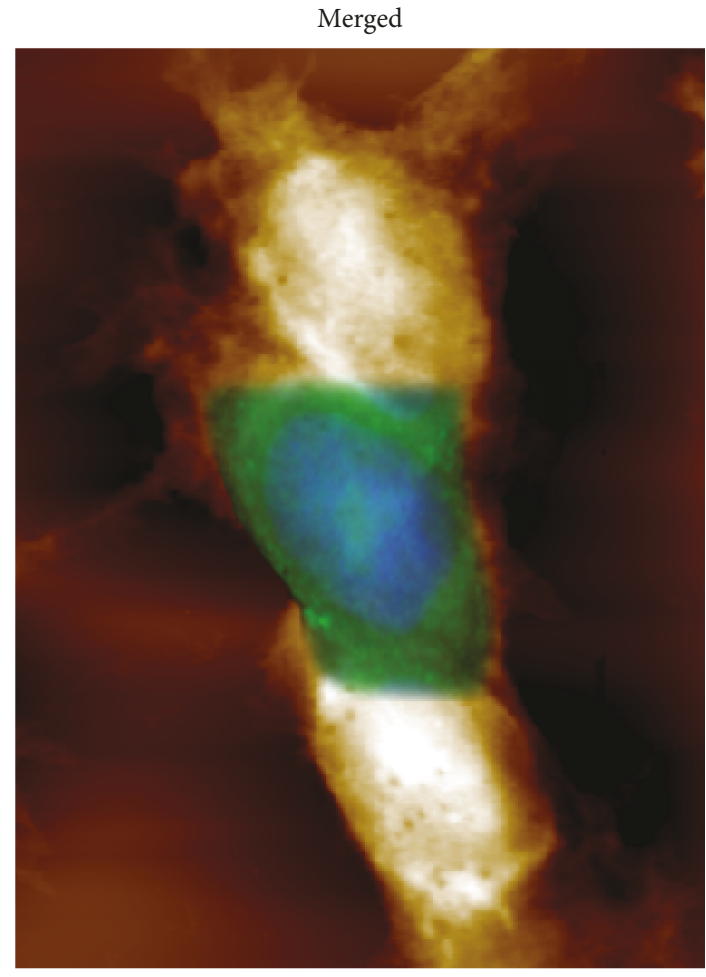

(d)

Figure 5: Combination of AFM with fluorescent microscopy: (a) MCF-7 breast cancer cells imaged with fluorescent microscopy (the blue color corresponds to DAPI staining that stains the cell nucleus and the green color represents antivasodilator-stimulated phosphoprotein (VASP) antibody. VASP is an actin-polymerization regulator which interacts with cell-ECM adhesion protein Migfilin and regulates cell migration [237]), (b) fluorescent image of three MCF-7 cells, (c) AFM image of the same cells, and (d) merged fluorescent and AFM images (unpublished data obtained by Andreas Stylianou in Cancer Biophysics Laboratory with an Olympus BX53 fluorescent microscope equipment, an Olympus XM10 monochrome CCD camera (1.4 megapixels), an UPlanFL N microscope objective lenses (40x/0.75 and 100x/1.30 oil), and a Molecular Imaging-Agilent PicoPlus AFM system (now known as 5500 AFM, Keysight Technologies)).

imaging can reveal the localization and quantify intracellular molecules and functions even at the level of a single molecule. Using specific fluorescence labelling, images of molecular mechanisms of biological functions can be acquired with high temporal resolution. Also, due to fluorescence intrinsic sensitivity to local microenvironment, valuable information on molecular specificities of cell structure can be obtained. However, as fluorescence imaging spatial resolution is limited by diffraction, its combination with AFM can produce images and provide us with information with high spatiotemporal resolution and biochemical specificity.

Generally, the combination of AFM with other microscopic imaging modalities can produce high-quality scientific information that cannot be achieved by using just one microscope.

3.2. AFM and Optical Microscopic Imaging. Optical microscopic techniques are often used in order to gain access to complementary information to AFM studies [45-47]. Using microscopies that utilize light in order to acquire images, such as simple optical, fluorescent, and laser scanning confocal microscope (CLSM), valuable information can be obtained concerning cellular process and interactions. Fluorescence microscopy incorporates fluorescent probes that either are accumulated in cellular components or are highly sensitive to environmental changes such as alterations of calcium ions and hydrogen ions. Because of its intrinsic sensitivity, fluorescence microscopy can image even a single fluorescent molecule if the background is not fluorescent [48].

CLSM uses a pinhole approach to spatially limit out-offocus light or glare from reaching the image detector. In that way, only the image of the focal plane is acquired providing better spatial resolution not only in $x-y$ plane but also across the $z$-axis $[49,50]$. Furthermore, by incorporating machine vision techniques and with the appropriate calibration, not only qualitative but also quantitative data are obtained [51].

In recent years, an increase in the number of studies that combine AFM with optical microscopic techniques has been published. The combination of the two modalities can provide unique information from a single cell (Figure 5) to a tissue level. Staunton et al. have developed a novel technique that combines AFM with a confocal microscope in order to explore the mechanical properties of soft heterogeneous three-dimensional samples [52]. They used metastatic breast 
adenocarcinoma cells invading into collagen hydrogels, determining the elastic properties of both the cell and the extracellular matrix. Cells that are actively invading or fully embedded into the collagen matrix were found to be stiffer than cells lying on the matrix surface. This stiffening is Rhoassociated protein kinase (ROCK) dependent, indicating that actomyosin contractility is a key player in the first phase of metastasis. On softer gels, enhanced invasion was observed either due to matrix softening by invading cells as a result of active rearrangement or proteolysis of adjacent fibers or because intrinsically softer tissues are preferable for invasion.

Stylianou et al. combined information from AFM with an optical and fluorescent microscope to study the tumor microenvironment such as fibroblasts (FBs) and cancer-associated fibroblasts (CAFs) [53]. In pancreatic tumors, treatment is reported to be blocked by desmoplasia, a cancer-specific type of fibrosis. Desmoplasia is a result of the interaction between stromal cells and tumor microenvironment. According to their results, CAFs express myofibroblast characteristics, such as $\alpha$-smooth muscle actin expression, cell elongation, and lamellipodia formation, and are softer than FBs. Transforming growth factor beta (TGF- $\beta$ ) increases cell stiffness (Young's modulus) of both types of fibroblasts and elongation, cell spreading, lamellipodia formation, and spheroid invasion only in CAFs. In another study, Cazaux et al. have used AFM to mechanically stimulate, in a controlled manner over time and space, single immune cells and fluorescence imaging to follow the consequences of the contact through calcium fluorescent probes [54]. But they have also used light through a photoactivatable small $G$ protein, Rac protein, to stimulate single $\mathrm{T}$ cell while recording cellular mechanical response. Canale et al. presented a review article trying to reveal the toxic interplay between lipid membranes and pathological protein aggregation in neurodegenerative disorders such as Alzheimer's and Parkinson's disease based on AFM studies [55]. In the study of Wegmann et al., neuroblastoma cells (N2a) and scrapie-infected mouse neuroblastoma $(\mathrm{ScN} 2 \mathrm{a})$ cells were studied with both AFM and immunofluorescence microscopy [56]. AFM imaging revealed a smooth surface topography of normal cells while scrapie-infected cells presented fibrillar aggregates on their surface. Immunofluorescence imaging, using a cellular prion protein ( $\mathrm{PrP}$ ) antibody (anti-PrP), revealed that $\operatorname{PrP}$ is homogeneously distributed in normal cells while it tended to segregate in preferential domains in ScN2a cells. Combining surface and fluorescence information, the authors concluded that the aggregated signal in SCNa2 cells is due to the structural changes at the cell surface. Finally, Kuyukina et al. using a hybrid imaging microscope, consisted of AFM and CLSM, studied bacterial viability at a single-cell level [57]. Fluorescent microscopy revealed that bacteria had compromised cell membranes when they were exposed to different organic solvent stresses. Combined information showed that changes in size, surface/volume ratio, and roughness were possible resistance mechanisms.

3.3. AFM and Second Harmonic Generation (SHG) Imaging. Except for fluorescence imaging, very promising microscopic modalities for biological imaging are based on nonlinear optics. These microscopic techniques can provide high- resolution, three-dimensional images without the use of fluorescent labelling. In that way, structural and dynamic images of living cells are acquired in a noninvasive manner. Among them, second harmonic generation (SHG) has been used in combination with AFM for investigation of biological samples. SHG occurs when two photons of the frequency $\omega$ interact with a nonlinear medium and are converted into a new photon with twice the energy $(2 \omega)$. As a nonlinear process, only noncentrosymmetric structures such as collagen are able to emit SHG signals [58]. Stylianou et al. proposed the combined AFM and SHG information as a valuable tool to understand the nonlinear optical properties of collagen and in the long-term SHG to be used as a nondestructive imaging modality to monitor collagen-related diseases [59]. They have developed thin collagen films with controlled characteristics that can help in modelling the collagen matrix in a variety of pathological conditions such as thermal injury or mimic the structure of the collagen matrix in different tissues and conditions. AFM was used for thin film characterization while the SHG signal was correlated and interpreted with the predetermined characteristics of the films. Navab et al. investigated integrin $\alpha 11 \beta 1$, a stromal cell-specific receptor for fibrillar collagens that is overexpressed in CAFs, as a regulator of cancer stromal stiffness and promoter of tumorigenesis and metastasis in non-small-cell lung cancer [60]. Images acquired with SHG signals provided information on the quantification of collagen organization while AFM provided measurements of Young's modulus of the cancer-associated stroma. Their findings showed that the $\alpha 11$ signaling pathway in CAFs is closely related with collagen cross-linking, with stiffness and with organization of fibrillar collagen matrices. In another study, Robinson et al. performed a quantitative analysis of 3D extracellular matrix (ECM) remodelling that is involved in a large number of physiopathological processes in biology [61]. Pancreatic stellate cell interactions with ECM were studied in a 3D environment via ECM remodelling. AFM studies provided collagen topography, mechanical properties, and stiffness, while SHG microscopy in combination with image analysis techniques was used to observe and acquire images of collagen structure and topology and to further quantify collagen fiber changes in terms of alignment and thickness. Their results demonstrated that the increased ECM stiffness observed in fibrosis can be correlated with ECM remodelling. Also, Chevalier et al. worked with enteric neural crest cells (ENCCs) in vitro and during colonization of the gastrointestinal tract in chicken and mouse embryos [62]. They used AFM to study the influence of the stiffness of the environment on ENCCs both in vitro and in vivo and SHG to study its structure. The AFM results revealed that although initially the mesenchyme of the gut was soft, its stiffness was gradually increased during ENCC colonization. Complementary to these findings, SHG showed a gradual organization and enrichment of collagen fibers in the developing gut that are associated with the abovementioned stiffness. Furthermore, Liu et al. suggested the use of a cell-based system to generate a mechanoresponsive cell system (MRCS) that responds specifically to mechanoenvironmental cues to sense and kill cancer metastasis [63]. SHG imaging reported high collagen 
expression colocalized with cancer metastasis. Collagen networks were observed to be more linearized in cancer regions than in noncancer ones. AFM revealed an increased stiffness in cancer regions that are heterogeneous. Combination of AFM and SHG findings suggest a strong correlation between collagen cross-linking and increased tissue stiffness at the metastatic sites indicating the presence of a distinctive cancer mechanoenvironment.

3.4. AFM and Scanning Electron Microscopy (SEM) Imaging. AFM and SEM are two of the most commonly used nonoptical scanning microscopes that can provide us with surface imaging and characterization with high resolution. SEM has an excellent depth of field while AFM has poor depth of field, but excellent contrast on flat samples. SEM is able to measure the chemical composition of surface features, and AFM can measure its physical properties. If only image acquisition time is taken into account, SEM is faster than AFM but it produces $2 \mathrm{D}$ images while AFM can provide us directly with sample height information.

In most of the correlative microscopy research, more detailed information can be obtained by exploiting the advantages of both SEM and AFM [64-66]. For example, Mahmoud et al. performed a comparative study between AFM and SEM on healthy human liver parenchyma [65]. Hepatic architecture was mapped using both imaging modalities. AFM visualized the cellular structure, nucleus, and chromatin granules whereas SEM revealed other structures not observed by AFM. Pavlova et al. used both AFM and SEM to highlight their diagnostic and screening capabilities in kidney cancer based on pathomorphological changes in patient's erythrocytes [66]. Kaul-Ghanekar et al. on another combined study investigated the cellular morphology along with surface roughness [67]. They explored the effect of the tumor suppressor protein SMAR1 on cell lines and on tumor sections of mouse and human breast cancer of different grades. SMAR1 was found out to be a significant modulator of surface roughness and cytoskeletal volume. Finally, they suggested that SMAR1 might be used as a phenotypic differentiation marker between healthy and cancer cells. Also, Iyer et al. found, using combined AFM and SEM, different brush layers on the surface of normal and cancerous human cervical epithelial cells [68]. These brush layers are important for interacting with the environment and can be taken into account in characterization of cancer cells by means of forces and mechanical parameters. In another study, Volakis et al. used a mouse xenograft model to study the effect of myoferlin, a protein involved in membrane dynamics, on tumor formation and local invasion [69]. SEM along with image analysis quantified cell surface area, as well as the number and length of lamellipodia and filopodia. AFM was used to image and determine Young's modulus, a measure of cell stiffness that was found to be reduced in myoferlin-deficient cells and the integrity of the cytoskeletal network.

\section{AFM and Collagen}

4.1. General. As it was mentioned in the previous sections, AFM provides the ability to acquire topographic images at the nanoscale combined to the mechanical property determination at specific regions. Hence, the topographical features of surfaces can be simultaneously acquired with its material stiffness. Novel AFM techniques, like bimodal AFM, have increased the nanoscale characterization capabilities of AFM to detect compositional variations in soft specimens, like proteins and cells [34]. Due to its high resolution, AFM has been widely used for the characterization of biomolecules such as fibrous and globular proteins. The main role of fibrous proteins is to provide mechanical stability [70]. Typical examples of fibrous proteins are the collagen and the amyloid fibrils, which are discussed in the next sections of this review.

AFM has been also extensively used for the mechanical characterization of globular proteins (i.e., proteins with a spherical shape and surface irregularities). Globular proteins present structural complexity [70], and its mechanical testing is a challenging procedure. Specifically, the classic models of applied mechanics, such as the Hertz model, provide several limitations in the analysis; thus, they can only be used approximately. Typical examples of globular proteins studied using AFM are the lysozyme, the bovine carbonic anhydrase II, and the lactate oxidase. In particular, Radmacher et al. investigated the viscoelastic properties of single lysozyme molecules (absorbed on mica). For the theoretical analysis of the results, they used the Hertz model and Young's modulus of lysozyme was determined to be equal to $0.5 \mathrm{GPa}$ [71]. In addition, for the investigation of the mechanical properties of bovine carbonic anhydrase II (BCA II), the modification of the Hertz model (which was proposed by Tatara [72]) was used. In the absence of Guanidine-HCl, Young's modulus of the native protein was determined to be equal to $75 \mathrm{MPa}$ [73]. The mechanical properties of lactate oxidase (which has applications in bioanalytical devices for lactate determination) were determined by analyzing the fitted data obtained from the force curves (provided by AFM) to the Hertz model for conical and spherical indenter geometries. Young's modulus values in the case of lactate oxidase were calculated in the range $0.5-0.8 \mathrm{GPa}[74]$.

4.2. Collagen. Collagens are the most abundant proteins in mammals and comprise almost the $30 \%$ of total cell protein in mammalian cells [75]. The collagen superfamily of vertebrate consists of more than 50 collagen and collagen-like proteins [76, 77]. The different members of the collagen superfamily have different shapes and sizes but the hallmark of a collagen is its monomer tropocollagen, which is composed of 3 polypeptide chains that form a right-handed triple-helical structure [76]. Each of these polypeptide chains contains a region with the repeating amino acid motif (Gly-X-Y), where $\mathrm{X}$ and $\mathrm{Y}$ can be any amino acid-12\% of tripeptides have sequence Gly-Pro-Hyp; charged residues like arginine, lysine, and aspartic/glutamic acid make up approximately 15-20\% of all residues in the monomer [78, 79].

The human body contains about 28 different collagens, among which fibrillar collagens and especially collagen type I are of most interest $[76,80]$. Collagen type $I$ is the major protein in the extracellular matrix [75], and it is characterized by unique properties such as self-assembly, biocompatibility, 


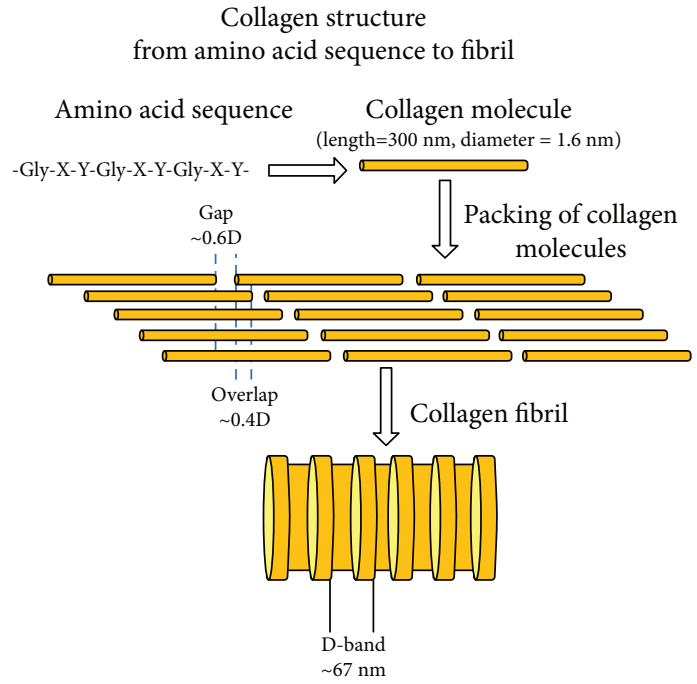

(a)

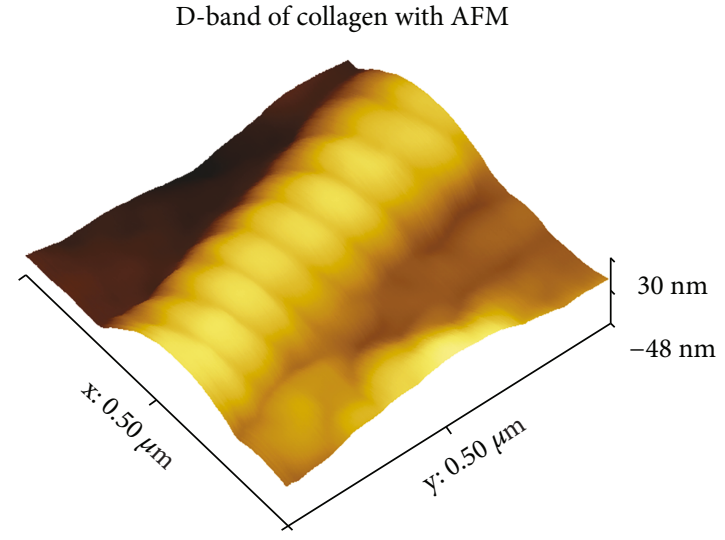

(b)

Figure 6: Collagen structure: (a) the collagen structure from the amino acid sequence to fibril. In this panel, the amino acid sequence, the collagen molecule, the packing of collagen molecules, and the structure of a collagen fibril are presented. (b) A collagen fiber D-band periodicity from a collagen thin film imaged using atomic force microscope (CPII Veeco-Bruker Microscope) in the tapping mode (adapted from [90]).

biodegradability, and nontoxicity [81]. Collagen type I molecules form rod-shaped triple helices assembled to form fibrils $[77,82]$, which are then properly aligned in order to form bundles and fibers [77, 82] (Figure 6(a)). Interestingly, the molecules are packed in a quarter-staggered fashion so as to form the D-band (also known as D-periodicity), which is a repeating banding pattern of about $67 \mathrm{~nm}$ (Figure 6(b)), depending on the tissue [75, 76, 83-86]. The fibrils of collagen type I are the elementary building blocks in many collagen-rich tissues $[87,88]$, while it presents different morphological functions (including tissue mechanical strength and scaffolding to cell migration) in different tissues [75, 77, 89]. Furthermore, collagen has been identified as a unique biomaterial for the formation of novel bioengineering interventions [75, 90-92]. AFM characterization (imaging and mechanical property measurements) has been extensively performed in pure collagen or collagen rich-tissues as AFM does not destroy the fibrillary structure of collagen and can offer information from collagen molecules to separated fibrils/fibers [81, 93]. AFM has been used for investigating a number of different issues concerning collagen, from collagen structure to collagen-related pathological conditions and collagen optical radiation or cell interactions.

The structural and the mechanical properties of collagen at the nanoscale under various conditions have been extensively studied using AFM $[5,22,90,94]$. The major advantage of AFM in collagen investigation comparing to other techniques is its ability to provide information regarding nanotopographical features such as the D-band periodicity (the collagen fibril consists of an alternating gap and overlapping regions with a highly reproducible $\mathrm{D}$-band periodicity of approximately $67 \mathrm{~nm}$ ) [17]. Simultaneously, AFM can provide data regarding the mechanical response of selected regions at the nanoscale. Hence, AFM is a suitable tool for the detection of local structural and mechanical variations of a single collagen fibril, under several external factors $[11,95]$. The unique ability to combine information regarding nanotopographical and nanomechanical features using AFM in real time has led to groundbreaking solutions for the investigation of collagen $[84,96]$.

Thus, AFM has been used in several investigation areas related to tissues which contain collagen and has been proven a valuable tool in the field of histology and cytology [97]. Furthermore, AFM has been used for the investigation of collagen fibril structural variations at the nanoscale which are related to various pathological issues such as diabetes [98]. In addition, AFM has been increasingly used in the investigation regarding the influence of radiations arising either from nature or from medical activities (e.g., UV irradiation, radiofrequencies) on tissues which contain collagen [99, 100]. For example, the influence of UV irradiation on collagen at the nanoscale is a crucial issue regarding the general populations' health, due the chronic exposure of human skin in sunlight. As a result, the information provided at the nanoscale using AFM techniques has opened new insights into collagen research and the correlation of collagen nanofeature alterations with pathological situations.

\subsubsection{Collagen-Ultraviolet (UV) Radiation Interactions.} The clarification of the interactions of skin with sunlight is of crucial importance since it is an intrinsic constituent of human life [101]. Solar radiation is made up of infrared, visible, and ultraviolet (UV-C, UV-B, and UV-A) radiation and exposure of the human body (i.e., skin, eyes) to sun and especially to UV can be harmful and lead to sunburn, photoaging, corneal damage, and carcinogenesis [102, 103]. Additionally, UV irradiation is applied for material science purposes, including for sterilizing and cross-linking biomaterials [104-106]. As UV can induce a number of alterations 
in collagen properties, including structure, chemical stability, and mechanical properties [107-111], AFM techniques have been extensively used for investigating UV-collagen interactions.

It has been shown that the irradiation of pure collagen with UV-C decreases the surface roughness [112-114], which is a crucial parameter in cell-surface crosstalk $[115,116]$ as modification in roughness alters the surface that is available for cell adhesion and growth [117]. The decrease of surface roughness due to UV irradiation has been demonstrated not only in pure collagen samples but also in collagen-based blends, including collagen-poly-vinyl alcohol, collagenpoly-vinyl pyrrolidone, and collagen-poly(e-caprolactone) $[112,118,119]$. Although surface roughness was modified through UV-C irradiation, it has been shown that for the same dose, collagen fibrils retained their characteristic D-band structure [114]. The D-band periodicity is important for the fibril mechanical properties and the cell-collagen crosstalk, while it has been correlated with specific pathological conditions $[84,86,120,121]$. Furthermore, AFM nanoindentation was used in order to investigate the effects of UV irradiation on the gap and overlapping zones of collagen D-band [17]. It was found that UV irradiation has a significant effect on the height level differences between the overlapping and gap zones, while the fibril stiffness (in terms of Young's modulus) in the D-band zone was reduced, which was associated with the UV-induced polypeptide chain scission. Moreover, it has been demonstrated that cells' behavior is affected when cells are cultured on UV-irradiated collagen-based substrates $[114,122,123]$. It has been shown that cells can normally spread on collagen-based substrates that were UV-irradiated with doses relevant to those that are used for sterilizing scope cells, but when the irradiation time was increased, the cells were characterized by abnormal growth (in terms or cell shape) [114]. In addition, it has been demonstrated that in some cases, UV irradiation of collagen supported cell growth and enhanced cell viability $[111,122,123]$. As AFM studies did not reveal significant modifications in topography when collagen is irradiated with low UV doses, the alterations in cell behavior have been suggested to be a consequence of either mechanical property alterations and/or chain scission $[100,124]$.

4.2.2. Low-Level Laser (LLL) Therapy Collagen Interactions. Low-level laser therapy (LLLT), or photobiomodulation [125], applies low-level (also referred and as low-power) lasers for the treatment of several pathological conditions or abnormalities, including fibromyalgia, osteoarthritis, tuberculosis, temporomandibular joint disorders, and wound healing that presents increased interest. In the case of wound healing, it has been suggested that the underling mechanism, which remains partially unknown, includes the increase of mitochondrial function, ATP, RNA, and protein synthesis [126]. Subsequently, these lead to increased oxygen consumption and enhanced synthesis of the reduced form of nicotinamide adenine dinucleotide (NADH) and ATP. As a result, a promotion of the cellular metabolism was observed along with enhanced wound healing mainly through the increase proliferation of fibroblasts and their ability to produce collagen. Although the collagen role in the progress has been identified, the LLL-collagen interactions remain partially understood. Stylianou and Yova in 2015 used AFM techniques in order to investigate the effects that LLL (irradiation in the red region, $661 \mathrm{~nm}$ ) has on collagen and the influence on cell culturing [127]. After LLLT irradiation, no statistical significant alterations were found in the nanoscale topographical characteristics of the collagen substrates (collagen thin films), but a negative effect (abnormal cell shape) was found on the fibroblasts that were cultured on the irradiated collagen substrates. As the modulation of fibroblast shape and behavior was recorded in the absence of measurable surface alterations, it was suggested that these effects are not influenced by the surface properties of the substrate through the surface contact guidance mechanism $[128,129]$. These results offer new insights toward the clarification of the LLLT mechanism and the role of collagen in this procedure.

4.2.3. Nanomechanical Properties of Collagen. Extended studies focused on collagen using AFM techniques have been performed during the last decades due to the fact that many parts of the mammalian body contain collagen in the form of fibers (e.g., the skin, bones, cartilage, and tendons contain collagen fibers). Typical measurements of collagen fibers have been performed by Minary-Jolandan and $\mathrm{Yu}$ [11]. In their research, the mechanical differences on collagen fibrils due to the D-band periodicity were revealed (Young's modulus values were calculated to be $\sim 1.2 \mathrm{GPa}$ for the gap and $\sim 2.2 \mathrm{GPa}$ for the overlapping regions). The mechanical heterogeneity due to the D-periodicity was also confirmed by Kontomaris et al. [17]. Mechanical differences in the periodic banding of collagen from a rat tail tendon was also reported by Grant et al. [84]. However, Grant et al. took both a static and dynamic indentation approach to show that the topography had variances not only in elastic moduli but also in the time-dependent behavior at physiologically relevant frequencies $(0.1-2 \mathrm{~Hz})$. In addition, Strasser et al. determined Young's modulus of collagen fibril type I in the range 1.2 $\pm 1 \mathrm{GPa}$ [130] and Yadavalli et al. in the range $1.03 \pm 0.31 \mathrm{GPa}$ [131]. In the same order of magnitude were the results presented in the research conducted by Heim et al. (i.e., Young's modulus values varied in the range $1-2 \mathrm{GPa}$ ) [132]. On the contrary, the results provided by Wenger et al. differ greatly comparing to the previous values (5-11.5 GPa) [133]. As mentioned, the key advantage of AFM is to carry out imaging and characterization under aqueous conditions, so it was shown that the nanomechancial properties of bovine Achilles tendon collagen fibrils changed by three orders of magnitude $(1.9 \pm 0.5 \mathrm{GPa}$ to $1.2 \pm 0.1 \mathrm{MPa}$ ) when going from ambient to buffer conditions [134]. Further, it was shown that reconstituted bovine Achilles tendon collagen can be mechanically tuned by altering the imaging buffer [78]. It was also shown that in imaging the same fibrils in air and under buffer, the diameter doubled in size. Then, the mechanical properties of collagen approximately doubled with addition of $1 \mathrm{M}$ monovalent chloride salts, further increased by reducing the $\mathrm{pH}$, and increased by a factor of 100 by testing under 100\% ethanol [78]. Most 


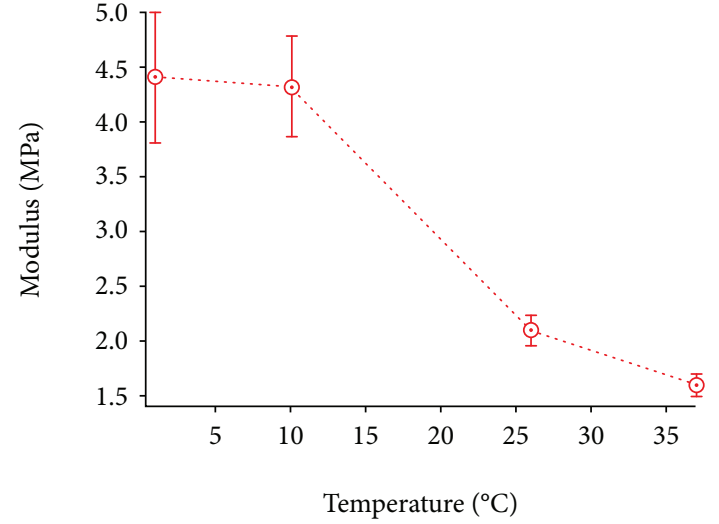

FIGURE 7: Moduli of a single individual collagen fibril at varying temperatures in PBS buffer (Colin Grant; unpublished data).

AFM studies test the compressive mechanical properties by nanoindentation; however, it is possible to carry out tensile testing on collagen fibrils [135]. Here, it was also shown that human collagen fibrils have reduced sensitivity to saline and $\mathrm{pH}$ compared to reconstituted collagen fibrils. As well as varying the aqueous conditions, the effect of temperature has also been carried out-nanomechanical properties of the same individual collagen fibril varied from $4.5 \mathrm{MPa}$ at $1^{\circ} \mathrm{C}$ to $1.6 \mathrm{MPa}$ at $37^{\circ} \mathrm{C}$ (Figure 7). This demonstrates the importance of carrying out nanomechanical property characterization of a biological matter under the most relevant physiological condition.

However, the determination of mechanical properties at the nanoscale is a challenging procedure. It is obvious from the results presented above that the extended range of values on Young's modulus values, even on the same sample, has been recorded (e.g., see the wide range of Young's modulus values regarding collagen in Table 1). The basic reasons for the wide range of Young's modulus values (even when the same sample is tested) are as follows:

(a) The restrictions which are provided by the classic models of applied mechanics, commonly used for the analysis of the data obtained using AFM

(b) The uncertainties in the experimental determination of critical magnitudes such as the spring's constant determination and the exact shape and dimensions of the AFM tip

(c) Different used protocols for the sample preparation (e.g., testing in air or in liquid environment)

An analytical discussion of the approximations regarding the AFM indentation technique on biological samples is presented in "Approximations of the AFM Nanoindentation regarding Biological Samples and Biomaterials at the Nanoscale" in Supplementary Materials (available here).

4.2.4. Collagen in Tissue Samples. As previously discussed, collagen is the most abundant protein in the human body and is found in most tissues that requires some form of biomechanical structure/strength. AFM analysis can be carried out to both image not only the collagenous structure of the tissue but also the nanomechanical properties. The sclera (white of the eye) has been investigated to show that the mechanical properties vary considerably on removing the episcleral layer to reveal the collagenous stromal layer [136]. The skin has the remarkable regenerative capacity to be able to heal itself in a complex cascade of biochemical and cellular remodeling processes. It was recently shown that the nanomechanical and viscoelastic properties of the upper dermis from the human skin change following wound healing [96]. Imaging revealed a variance in the orientation of collagen fibrils in scar tissue compared with health skin tissue, but also the scar tissue is stiffer than healthy tissue and also healthy tissue retains a higher dissipative characterization [96]. Similar dynamic/static nanoindentation was also carried out on porcine blood vessels [137]. The outer layer of the blood vessels (tunica adventitia) is a collagen layer that helps prevent overexpansion. Here, the adventitial layer of the porcine aorta vs. pulmonary arteries was compared, under aqueous conditions at $37^{\circ} \mathrm{C}$. It was demonstrated that the aorta exhibited larger viscoelastic and dissipative characteristics, which makes sense as the aorta is under higher pressure compared with the pulmonary artery [137].

\section{AFM and Specific Pathological Conditions}

\subsection{Articular Cartilage and Osteoarthritis}

5.1.1. Cartilage. Articular cartilage is a connective tissue, which provides wear-resistant joint motion and low friction. It covers the ends of long bones and its thickness is in the range $1-3 \mathrm{~mm}$. The main function of articular cartilage is to absorb and distribute the applied loads due to the joint movements. In addition, it provides protection to subchondral bone from damage. The functionality of articular cartilage is engendered by its collagen fiber meshwork and extrafibrillar proteoglycan-rich matrix which is highly hydrated [138]. Loss of collagens or proteoglycans or structural changes may lead to a water content change and is a result to several damages to the tissue. As it has been previously reported, a loss of proteoglycans from the hydrated gel which is followed by an irreversible collagenolytic degradation of the fibrils results in damages regarding the collagen meshwork and as a result to the development of osteoarthritic cartilage [139].

Various methods are able to provide direct in vitro observation of cartilage under physiological conditions. Typical examples are the optical microscopy [140, 141], visual inspection, and histology $[142,143]$. However, these methods are limited to a spatial resolution of approximately $200 \mathrm{~nm}$. In addition, in the case of electron microscopy, ultrastructural details of articular cartilage at a molecular level can be provided, but the procedure requires dehydration and chemical fixation of the cartilage combined with metal staining or sputtering. Thus, the cartilage specimen cannot be examined at near physiological conditions. Moreover, optical and electron microscopy cannot provide measurements of the mechanical properties of articular cartilage.

The most direct method for stiffness measurements is the classic compression testing. However, the compression test 
TABLE 1: Typical examples of Young's modulus values of collagen in the literature.

\begin{tabular}{lccc}
\hline \multirow{2}{*}{ References } & Collagen source & $\begin{array}{c}\text { Young's modulus on collagen } \\
\text { Contact model used }\end{array}$ & Young's modulus values (GPa) \\
\cline { 2 - 4 } & Type I collagen from bovine Achilles tendon & Oliver \& Pharr model & $0.74-1.43$ \\
{$[131]$} & Type I atelocollagen from calf skin & Hertz model & $1.03 \pm 0.31$ \\
{$[11]$} & Type I collagen from bovine Achilles tendon & Hertzian model, modified for cylindrical sample & $1.2-2.2$ \\
{$[133]$} & Type I collagen fibrils from rat tail tendons & Oliver \& Pharr model & $3.75-11.5$ \\
{$[130]$} & Type I collagen fibrils from calf skin & Hertz model & $1.2 \pm 1$ \\
\hline
\end{tabular}

requires sample manipulation (e.g., the sample's surfaces have to be highly parallel and the cartilage has to be cut off the bone). On the other hand, macroindentation procedure does not require sample manipulation; hence, typical indentation testing devices can be used to evaluate the quality of the health or the disease state of articular cartilage [144-146]. These devices usually employ indenters with diameters in the range 1-2 $\mathrm{mm}$ in order to evaluate the correspondence of the sample to a specific applied load. However, stiffness measurements at the millimeter scale are not sensitive to detect pathological conditions (e.g., early-stage osteoarthritis) or changes due to aging [144, 146, 147].

5.1.2. Osteoarthritis. As it was already mentioned, various methods have been used for the determination of cartilage's pathological conditions; however, they are limited to specific conditions (e.g., measurements at the microscale or measurements at nonphysiological conditions) or they provide only specific information (e.g., imaging or mechanical properties). On the contrary, AFM provides the possibility of simultaneous imaging and mechanical property measurements (i.e., Young's modulus maps) on a micro- or nanometer scale in specimens that are near physiological conditions. Articular cartilage presents an interesting behavior depending on the level that is being tested. This interesting behavior has been revealed using AFM. In particular, at the micrometer scale, it behaves as a uniform and non-structured material. Hence, at the microscale, it presents an overall stiffness value which can be used as an initial approximation in order to evaluate its properties. In addition, it must be noted that cartilage can be modeled as a poroviscoelastic material; hence, the overall stiffness measurements provide an aggregate modulus, both viscous and elastic. As it has been previously reported, the aggregate modulus ranges between the values $1 \mathrm{MPa}$ and $60 \mathrm{MPa}$ depending on the loading conditions $(\sim 1 \mathrm{MPa}$ for low-frequency conditions $(<0.1 \mathrm{~Hz})$ and $\sim 60 \mathrm{MPa}$ for high-frequency loading $(\sim 40 \mathrm{~Hz}))$.

Moreover, Young's modulus at specific regions of articular cartilage at the microscale or at the nanoscale can be calculated using AFM. As it has been previously reported, the elastic modulus of the articular cartilage intercellular matrix exhibits a depth-dependent increase. Specifically, Young's modulus value at the superficial zone is approximately $0.52 \mathrm{MPa}$ and at the calcifying deep zone $\sim 1.69 \mathrm{MPa}$ $[148,149]$. The major advantage of AFM comparing to traditional methods is that is able to provide information regarding the functionality of articular cartilage. Thus, using AFM, diseases such as osteoarthritis can be detected at the initial stages. AFM has been used by many researchers as a tool for the diagnosis of cartilage's pathological conditions at early stages [14, 15, 150]. In particular, Stolz et al. [15] calculated Young's modulus of normal articular cartilage at the microscale $(2.6 \mathrm{MPa})$ and nanoscale $(0.021 \mathrm{MPa})$. The results of Loparic et al. are in the same order of magnitude. The microstiffness of articular cartilage was determined to be $1.3 \pm 0.4 \mathrm{MPa}$ and the nanostiffness $22.3 \pm 1.5 \mathrm{kPa}$ for cartilage's the proteoglycan gel (PG gel) and $184 \pm 50 \mathrm{kPa}$ for the collagen meshwork [150]. Stolz et al. investigated osteoarthritis at early stages and proved that microstiffness of articular cartilage does not present differences comparing to healthy cartilage. Hence, osteoarthritis at the early stages can be only detected at the nanoscale. In particular, at the nanoscale, nanostiffness was calculated to be $83 \mathrm{kPa}$ in the case of normal cartilage and $5.6 \mathrm{kPa}$ in the case of grade 3 osteoarthritis [14]. Hence, these findings proved the ability to detect early stages of osteoarthritis using AFM and have opened a new prospect for the use of AFM as a clinical tool. In particular, Stolz et al. tested AFM in real clinical activities; articular cartilage biopsies from seven patients who suffer from osteoarthritis (the age of the patients was in the range 62-96 years) were examined [14]. The result of this research has revealed an increasing softening of articular cartilage at the nanoscale with progressing osteoarthritis at a given age. It must be noted that all the detected changes at the initial stages of osteoarthritis could only be detected at the nanoscale.

In addition, Stolz et al. have shown that biomechanical and morphological changes occurring in articular cartilage during normal aging differ comparing to the ones occurring during osteoarthritis progression. The cartilage softening during osteoarthritis progression is due to the disintegration of collagen meshwork [14]. The stiffening of the collagen meshwork at the nanoscale can only be detected using AFM [14]. Hence, the ability to detect changes at early stages of osteoarthritis and distinguish these changes from the ones that occurred due to normal aging has opened an exciting prospect of using AFM as a tool in real clinical activities.

5.2. Cancer. Cancer progression has been associated with alterations in cancer cells and tumor microenviroment (TME) components [151, 152]. Alterations in cancer cells that make them differ from normal cells include changes in cell morphology, reproduction, communication, adhesion, cell-to-cell or cell-to-ECM interactions, cell invasion/metastasis, and even cell death. Recently, it has been demonstrated that cancer cells have different nanomechanical properties 
than normal cells and alterations in the mechanical properties of cells play a crucial role during malignant transformation [152, 153]. TME consists of the tumor blood and lymphatic vessels, the stromal cells (FBs and CAFs), the ECM (including mainly collagen and hyaluronic acid), and a number of other soluble factors. Tumors have the tendency to modify their microenvironment in a way that promotes tumor growth and progression. For instance, in desmoplastic tumors, like pancreatic and breast cancers, the interactions between TME components lead to desmoplasia, which is characterized by the presence of CAFs and the overproduction of ECM components [154, 157]. This desmoplastic reaction leads to TME stiffening which increases the compressive mechanical forces in the tumor interior $[113,155,156]$. Furthermore, the crosstalk between TME and cancer cells influences a number of cancer cells' properties, including cell proliferation, migration, and cell invasion through surrounding tissues. Consequently, the understanding of cancer cells and TME alterations during cancer progressions is of utmost importance, in order to develop novel and more effective anticancer therapies or diagnostic techniques. There are several studies correlating the influence of the mechanical properties of the ECM and tumor progression [157, 158]. For instance, it has been demonstrated that caveolin-1 (Cav1, the major component of endocytic caveola plasma membrane, promotes among others the force-dependent contraction and TME stiffening [158]. AFM arises as a key tool in this research area, providing many novel research results $[4,159,160]$. AFM can be used as a unique technique in order to study cancer cell properties (such as growth, invasion, and metastasis), TME alterations, and cancer tissue progression. Generally, its ability to work on live cells with high resolution and to assess nanomechanical properties (e.g., Young's modulus properties) has made AFM a valuable technique in the field of cancer research. Apart from in vitro experiments of AFM on cancer cells, AFM has been used for studying the nanomechanical properties of TME components [4] and cancer tissue biopsies with great potentials [160-162]. In this section, we first present the use of AFM for characterizing the nanomechanical properties of cancer cells and its use for discrimination between healthy and cancer cells. Then, we discuss the use of AFM for investigating the nanomechanical properties from tissue biopsies and the possible application for early cancer diagnosis.

\subsubsection{Cancer Cells}

(1) High-Resolution Imaging. Although it has been demonstrated that cancer cells have different characteristics and properties than normal cells, they remain poorly identified or understood [163]. AFM techniques have already been used for studying cancer and normal cells in terms of morphology, cells' surface, pericellular activity, proteolytic activity, exosomes, cell-cell or cell-ECM interactions, and nanomechanical properties.

One of the key characteristics of AFM and one of its major advantages are its ability of high-resolution imaging. Although other techniques, like optical and fluorescence microscopy, can offer significant information concerning cancer cell morphology at the single-cell level, AFM can provide information at the nanoscale concerning cell morphology and surface. For instance, AFM has been used for studying invadopodia which are cellular processes extended by the cells and play a significant role in cancer cell invasion and metastasis. Chasiotis et al. and Fillmore et al. used AFM to image the surface of human glioblastoma brain tumor cells (T98) on collagen substrates during invasion process $[164,165]$. They demonstrated that invadopodia present an unusual nanomorphology and their results offered new insights concerning cell-substrate interactions. Additionally, AFM high-resolution imaging has been used for the characterization of single exosomes [166], which play a significant role in tumor proliferation and tumor microenvironment modulation [167-169]. Sharma et al. applied AFM techniques in order to investigate the exosomes from normal and cancer cells and showed that cancer cell-derived exosomes possess surface nanofilaments that may help in cellcell communication [170]. Also, AFM can be applied to investigate specific cell activities that seem to be crucial for cell metastasis, as pericellular proteolytic activity which is important for TME remodeling and cancer cell invasion [171]. AFM can be used for assessing differences in average height, volume, and molecular weight distribution of pericellular matrix proteins in TME [171-173].

In the research area of cell-substrate interactions, AFM can provide unique information concerning the interactions of normal and cancer cells with culture substrates and obtain new data about cell-ECM interactions. It has been demonstrated that nonmetastatic breast cells adhered less to mineralized ECM secreted by osteoblasts than metastatic cells [174]. Furthermore, AFM has been used for investigating the mechanical response of cancer cells on different substrates. Park et al. studied prostate and breast cancer cells that were cultured on nanoscafolds and showed that different cancer cell lines have distinct response to substrate characteristics such as size and nanomechanical properties [175]. Additionally, AFM can be used for studying cell-cell interaction. For instance, Puech et al. studied melanoma cell line during the binding process on human umbilical vein endothelial cells [176], while Laurent et al. investigated the adhesion strength between endothelial cells and cancer cells and demonstrated that the more invasive cells formed the strongest bonds with endothelial cells [177]. Hoffmann et al. used AFM in order to study the interaction forces among tumor cells and natural killer cells and demonstrated that in order to separate natural killer cells from cancer cells, the required forces are higher when the killer cell receptor $2 \mathrm{~B} 4$ is activated [178].

(2) Mechanical Properties of Cancer Cells. Another area that presents a significant research interest is the use of AFM for assessing cancer cell nanomechanical properties. It can provide extremely high precision on the force applied to the cell, live and fixed ones, under natural conditions (e.g., in liquid conditions with temperature control), and it can provide quantitative mechanical measurements simultaneously to high-resolution imaging. As alteration in cytoarchitecture is 
Elasticity profiles
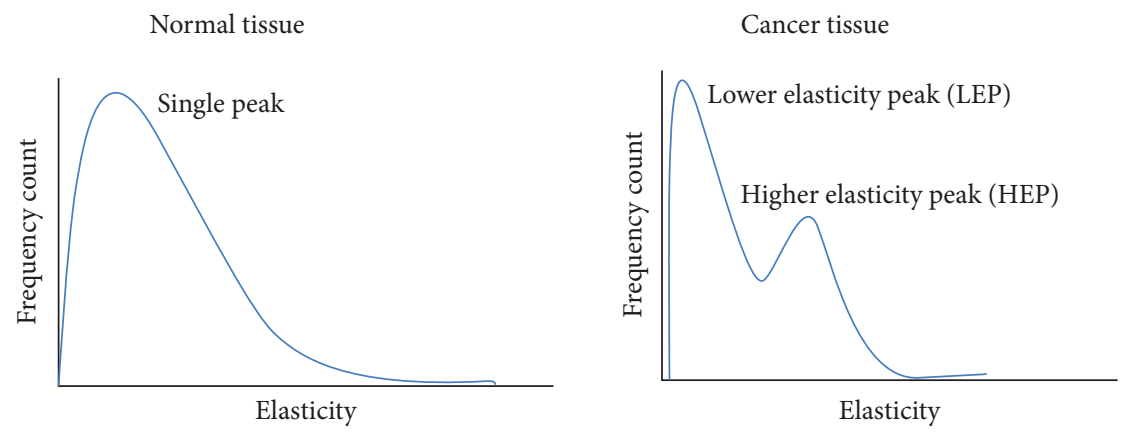

FIGURE 8: Schematic representations of predicted AFM elasticity measurements in normal and cancer tissues.

associated with cancer progression $[164,179,180]$ and the cells' mechanical properties play a crucial role in cell motility, metastasis, and growth [181, 182], the investigation of the nanomechanical properties of cancer cells, in comparison with normal ones, is of particular scientific interest.

One of the first demonstrations of the AFM mechanical measurements on cancer cells was performed in 1993 by Weisenhorn et al. on lung cancer cells, but with a quite big variation in their Young modulus values. Afterwards, forcevolume mode was used in order to provide enough statistics on embryonic cancer cells by Goldmann et al. [183, 184]. Although the study of single cancer cells can offer significant information concerning their mechanical properties, in 1999, the pioneer work of Lekka et al. demonstrated that the comparative investigation of cancer and normal cells can provide unique data $[185,186]$. Bladder cells with different levels of malignancy were used, and the results demonstrated that cancer cells were softer than normal cells. This was the first study that demonstrated that AFM can be used for distinguishing cancer cells from normal cells in terms of cell deformability. In order to further correlate the cell malignancy with their deformability, a number of studies have been performed on different cell lines, including breast $[161,187]$, prostate $[161,188]$, and oral [189] tumor cells, showing that cancer cells are softer than normal cells in terms of Young's modulus values. Furthermore, Cross et al. investigated cells that were obtained from pleural effusion of patients and showed that metastatic cells are softer than benign cells in clinical samples [190], while in a number of studies, it has been demonstrated that cells derived from normal or nonmalignant tissues present a similar pattern [153]. All these studies suggest that the stiffness of tumor cells (in terms of Young's modulus) can be considered as a diagnostic method for distinguishing them from normal cells [161]. Additionally, recently, it was demonstrated that CAFs were also softer than normal FBs and present increased invasive properties [53]. Furthermore, it has been demonstrated than malign and benign cell lines present different viscoelastic properties [43]. More specifically, cancer cells are characterized a substantially larger loss tangent than benign cells [43]. On the other hand, it has been suggested that cell softening is a cell-type-specific response [191] and that this cell characteristic alone fails to serve as a universal indicator for metastatic progression [192]. Consequently, more research is demanded toward this direction so as AFM to be used as a diagnostic tool for detecting cancer cells and distinguish them from normal ones in real clinical practice.

5.2.2. Cancer Tissue. Although in the previous section we present that cancer cells are softer than normal cells, it is a common belief that tumors are stiffer than their host tissue [162]. Tumor stiffening is caused by the increase in ECM composition, mainly collagen type I and collagen crosslinking during cancer progression [193-195], and it is the major biomechanical property of solid tumors that clinicians and patients can feel during palpation. Concerning the mechanical properties of tumor, AFM seems to be a valuable tool for assessing its nanomechanical properties with high spatial resolution. Although it has been presented that AFM has the potentials to be a unique cancer diagnostic tool $[162,180,196]$, the work toward this direction is limited [160, 197-199].

One of the first studies on this area was performed on snap-frozen mammary tissues, and the results demonstrated that the malignant epithelium was stiffer than in isolated breast cancer cells and that the ECM adjacent to the epithelium progressively stiffens [198]. In 2012, Lekka et al. investigated tissue samples from patients with endometrioid carcinoma of the uterine, breast, and vulvar cancers and their results demonstrated that these tissues were softer than the nonneoplastic regions [161]. In a pioneer work, in the same year, Plodinec et al. demonstrated that AFM can be used for assessing unique nanomechanical fingerprints from human breast biopsies that can be used for cancer breast diagnosis [162]. Their results revealed that normal and benign tissues present a uniform stiffness (in terms of Young's modulus) distribution, while malignant tissue was characterized by two distinguishable maxima. The same maxima were reported by Tian et al. in 2015 in human liver tissue and were referred as lower elasticity peak (LEP) and higher elasticity peak (HEP) (Figure 8) [199]. Also, in this study, the different stiffness distributions and maxima of other tissues (including esophageal cancer, clear cell renal cell carcinoma, colon cancer, and papillary renal cell carcinoma) were reported.

In 2016, Ciasca et al. demonstrated that AFM can assess nanomechanical fingerprints of human glioblastoma and 
meningothelial meningioma and AFM techniques can be applied for tumor brain grading [200]. In the same year, Ansardamavandi et al. developed clustering algorithms in order to divide and categorize the derived stiffness (in terms of Young's modulus values) measurements [197]. With their technique, they achieve to divide the measurements into three categories (the cellular, the noncellular, and the fibrous part of the tissue) and demonstrated that AFM can be combined with computational methods for identifying more than two different components of the tissue. In 2017, Cui et al. used AFM for investigating the nanomechanical fingerprints of cervical cancer and cervical intraepithelial neoplasia [201]. The research results demonstrated that both healthy and cancer samples present bimodal distribution of Young's modulus values with similar values of LEP, but cancer HEP values demonstrated that cancer tissues were significantly stiffer than the healthy controls. Consequently, HEP values could be used as a nanomechanical biomarker for cancer diagnosis. Also, in 2017, Minelli et al. applied an operator-independent neural network in order to identify the nanomechanical fingerprints of brain cancer [202]. Their technique achieves to identify and distinguish cancer from healthy tissue in a fully automated fashion.

The results so far have demonstrated that AFM is arising a novel technique for assessing the nanomechanical properties of cancer tissue and has the potential to provide a novel tool for early cancer diagnosis.

\subsection{Alzheimer's Disease}

5.3.1. Amyloid Fibrils. The study of protein fibrils is an important subject in various research fields and disciplines. A very interesting fibrous protein complex is amyloid fibrils which are highly ordered fibrillar structures assembled from either peptides or unfolded proteins [203]. The deposition of amyloid, in the form of amyloid plaques, has been associated with a number of degenerative diseases, while the amyloid structure has also been found in many functional proteins that are not related with a specific disease. As the involved mechanisms remain unknown, the clarification of the mechanisms of fibrillation, the structural features of the amyloid fibrils, and their physical and nanomechanical properties will help to reveal their biological role [203]. AFM and its capabilities for nanomechanical characterization of biological samples arise as a very significant tool for the study of the amyloid fibrils. For instance, Roeters et al. used AFM in combination with other techniques (such as $\mathrm{X}$-ray powder diffraction and IR spectroscopy) for studying the aggregation of the intrinsically disordered protein alpha-synuclein $(\alpha S)$ into amyloid fibrils [204]. These amyloid fibrils have been correlated with the pathology of Parkinson's disease. Their results demonstrated that the structure of $\alpha \mathrm{S}$ fibrils varies as a function of ionic strength, and they argued that this sensitivity to the ionic strength might form the basis of differences in $\alpha \mathrm{S}$-related pathologies. Also, in the last years, many researchers have focused on the self-assembly of amyloid peptides and proteins at interfaces since it will provide significant information for understanding the mechanism of some neurodegenerative diseases
[205]. The AFM has been employed in this area and so far, the results demonstrate that interfaces play an important role in peptide assembly [205]. Furthermore, very recently, Watanabe-Nakayama and Ono used high-speed AFM (HS-AFM), which allows the video imaging of the conformational changes of individual molecules, in order to investigate the structural dynamics of individual amyloidogenic protein assemblies [206].

5.3.2. Alzheimer's Disease. As it was already mentioned, the formation of fibrous particles is associated with a number of specific diseases. One of these, which have a great social impact, is Alzheimer's disease (AD). Patients with this disease presents neuritic plaques and vascular deposits consisting from fibrous aggregates of the protein amyloid- $\beta(\mathrm{A} \beta)$. More specifically, the hydrophilic molecules of $\mathrm{A} \beta$ accumulated outside of the neural cells and result in the formation of these highly toxicity amyloid plaques [207]. These amyloid plaques are composed of amyloid fibrils and small oligomers, which are insoluble protein aggregates [208]. Although the aggregation of the $\mathrm{A} \beta$ protein has been correlated with neurotoxicity, the involved mechanisms remain unclear [7]. Soon after the development of the AFM, it was demonstrated that the AFM is a powerful tool for studying the $\mathrm{A} \beta$ amyloids and the AFM can provide unique information for understanding the structural origins of this complex neurodegenerative disease [7]. For instance, in 1996, AFM was used to investigate the self-assembly and the surface structure of the resultant fibrils $[209,210]$. AFM has been applied in many studies since then for studying $\mathrm{A} \beta$ amyloids with several research purposes. Some of the more recent research results use advanced, sophisticated, and coupled AFM techniques. Furthermore, AFM has been used for studying $\beta$ amyloid structure in liquid conditions [211] and $\beta$-amyloid aggregation as a function of concentration [212]. Also, Connelly et al. investigated the ion channel mechanism and pore structures of the amyloids of AD pathology by using AFM [213], while several studies study the relationship between the formation of $\beta$-amyloid fibrils and toxicity in AD [214]. Furthermore, Song et al. used AFM in combination with fluorescence spectroscopy so as to study the interactions between vanillin and $\mathrm{A} \beta$ polypeptide [215]. Their results demonstrated the depolymerization of $\mathrm{A} \beta 1-42$ aggregates by vanillin in a dose-dependent manner, and the authors suggested that vanillin may be a potential pharmacological agent for the treatment of AD. In another study, Han et al. developed a sophisticated AFM-based technique to evaluate the amyloid precursor protein (APP) cleavage mechanism at the nanomolecular level, as the clarification of the APP cleavage mechanism is crucial for the development of new $\mathrm{AD}$ therapeutic agents [216]. Also, Banerjee et al. studied isolated cross-linked $\mathrm{A} \beta 42$ trimers ( $\mathrm{A} \beta$ oligomers) with HS-AFM which enabled the visualization of the structural dynamics of the oligomers at nanometer resolution on a millisecond time scale. According to the researchers, the nanoscale characterization of the $\mathrm{A} \beta$ oligomer structure and dynamics can lead to the development of novel oligomerspecific therapeutic agents. In other recent works, Li et al. used real-time AFM combined with molecular dynamics 
simulations as a novel approach to investigate the amyloid nanostructures formed by a potential pentapeptide inhibitor [217], while Han et al. applied AFM in order to probe the interactions between $\mathrm{A} \beta$ and two kinds of antibodies [207].

\subsection{Viruses and HIV}

5.4.1. Viruses. AFM has opened a new prospect regarding the investigation of the physical properties of viruses [218, 219]. The research regarding the structural properties of viruses and virus mechanics facilitates the engineering of their physical properties in order to improve their applications in bionanotechnology and molecular biology [220]. In particular, AFM provides the ability to acquire topographic images of biological objects at the nanometer scale (such as the viruses). The first imaging attempt regarding viruses, using scanning probe microscopy (STM), was provided by Baró et al. [221]. The virus particle that was visualized was the bacteriophage Ф29. But as in STM imaging the sample has to be electrical conductive, the particle was covered with a metallic layer that is far from its physiological conditions. This limitation has been overcome using AFM, which does not require electrical conductivity. AFM has been widely used for the topographic property determination of viruses, partially disassembled viruses, viral capsids, nucleic acids, etc. [222-225]. In addition, AFM can be used in order to investigate the viral infection in live cell process [226-229] and for the determination of the interaction forces between viruses and other molecules [220].

Viruses are solid-state objects; thus, the determination of their mechanical properties is possible. For almost all "empty" capsids, Young's modulus has been determined under the assumption that the capsid is homogeneous and hollow with idealized geometry [220]. In addition, the shell thickness and the empty capsid's size are considered to be similar to the real capsid's size $[218,220]$. For Young's modulus determination, the thin elasticity theory and finite element analysis on the model particle are usually used. However, the validity of this approach for Young's modulus determination is under discussion [230]. A different approach for the determination of the mechanical properties of viruses is based on the consideration of the virus particle and the AFM cantilever as two ideal springs in series. In this case, the "spring constant" of the particle $k_{p}$ can be determined using the following equation: $k_{\mathrm{ef}}^{-1}=k_{p}^{-1}+k_{c}^{-1}$ where, $k_{\mathrm{ef}}$ is the value of the effective spring's constant (which is calculated from the slope of the force vs. distance curves) and $k_{c}$ is the cantilever's spring constant. However, despite the fact that stiffness, as it is calculated using the spring constant, is an object's property, it does not depend only on the material but also depends on the object's dimensions and geometry.

Moreover, an alternative method for the mechanical property determination of viruses is the structural strength determination. In the most of the cases, the linearity of the force vs. distance curves is lost when a specific force is applied. The loss of the linearity is due to the mechanical failure or buckling in the particle. In addition, it must be noted that for the mechanical property determination of particles, a number of load-indentation curves are used. The force experiments usually do not cause permanent damages to the particles. However, a big number of indentation experiments may result in a nonlinear mechanical response or in mechanical failure. This behavior is caused by the material fatigue of the particle.

5.4.2. HIV. A typical example of a virus in medicine, with great significance and social impact that can be tested using the AFM methods, is the human immunodeficiency virus (HIV). HIV is an enveloped retrovirus with a genetic material that in the first phases of development is in a single-stranded RNA form [231]. In the mature phase of development, the viral genomic RNA is encapsidated within a cone-shaped capsid [231]. HIV capsid is a conical shell with a length $\sim 100-120 \mathrm{~nm}$ which is formed during viral maturation by the assembly of about 1500 capsid protein molecules (these molecules are organized into 250 hexamers and 12 pentamers approximately). The presence of these pentamers induces the curvature necessary to form the cone shape of the capsid [232-234].

The calculation of the stiffness of the capsid is mandatory for the assessment of any mechanical model regarding the capsid's uncoating. Several experiments for the determination of the mechanical properties of HIV 1 particles using AFM have been performed during the last decade $[220,231,235,236]$. In particular, Ramalho et al. applied the nanoindentation technique to measure the stiffness of empty capsids independently from the viral envelope. In particular, in vitro assemblies of wild-type and mutant recombinant HIV-1 capsid protein and isolated and mutant HIV-1 cores (i.e., filled capsids) were tested. This research resulted that the mutant assemblies were significantly stiffer than the wild-type assemblies. Pang et al. investigated the mechanism under which the physical properties of viral particles change during maturation and the effects of these changes in the viral lifestyle [235]. Their results indicated that HIV presents a severe reduction in particle stiffness during maturation that is mediated by the viral envelope protein. Kol et al. compared the mechanical properties of mature and immature HIV viruses using AFM nanoindentation [236]. The spring constant of the mature HIV-1 virus was calculated equal to $0.22 \mathrm{~N} / \mathrm{m}$ while the spring constant of immature HIV-1 virus was calculated equal to $3.15 \mathrm{~N} / \mathrm{m}$. In addition, Young's moduli were found to be $0.44 \mathrm{GPa}$ and $0.93 \mathrm{GPa}$ for the mature and the immature HIV-1, respectively, which means that the immature HIV-1 virus is stiffer than the mature one. The research regarding HIV is of great importance due to the fact that the mechanical softening as a result of maturation of HIV-1 (which is due to the loss of Env-Gag interactions) is probably a necessary condition before the infection occurrence [220].

\section{Conclusions}

AFM is a unique tool for nanocharacterization, including high-resolution imaging and nanomechanical measurements, of biological samples under different environmental conditions. New advances of AFM enable the simultaneous imaging with other modalities offering new perspectives in 
the field. A plethora of studies so far have demonstrated the abilities of AFM for assessing unique nanocharacteristics of biological samples that can be correlated with different pathological conditions. Consequently, AFM stands out as the ideal research instrument for exploring the detection of pathological conditions even at very early stages, making it very attractive in the area of bio- and nanomedicine.

\section{Abbreviations}

$\begin{array}{ll}\text { 3D: } & \text { Three dimensional } \\ \text { AD: } & \text { Alzheimer's disease } \\ \text { AFM: } & \text { Atomic force microscopy } \\ \text { APP: } & \text { Amyloid precursor protein } \\ \text { ATP: } & \text { Adenosine triphosphate } \\ \text { CAFs: } & \text { Cancer-associated fibroblasts } \\ \text { CLSM: } & \text { Laser scanning confocal microscope } \\ \text { ECM: } & \text { Extracellular matrix } \\ \text { ENCCs: } & \text { Enteric neural crest cells } \\ \text { FBs: } & \text { Fibroblasts } \\ \text { HIV: } & \text { Human immunodeficiency virus } \\ \text { HS-AFM: } & \text { High-speed AFM } \\ \text { ILK: } & \text { Integrin-linked kinase } \\ \text { KFM: } & \text { Kelvin force microscopy } \\ \text { LLLT: } & \text { Low-level laser therapy } \\ \text { MRCS: } & \text { Mechanoresponsive cell system } \\ \text { NADH: } & \text { Reduced form of nicotinamide adenine } \\ & \text { dinucleotide } \\ \text { PrP: } & \text { Prion protein } \\ \text { SEM: } & \text { Scanning electron microscopy } \\ \text { SHG: } & \text { Second harmonic generation } \\ \text { SPM: } & \text { Scanning probe microscopy } \\ \text { STM: } & \text { Scanning tunnelling microscopy } \\ \text { TEM: } & \text { Transmission electron microscopy } \\ \text { TME: } & \text { Tumor microenvironment } \\ \text { UV: } & \text { Ultraviolet } \\ \text { VASP: } & \text { Vasodilator-stimulated phosphoprotein. } \\ & \end{array}$

\section{Conflicts of Interest}

The authors declare that there is no conflict of interest regarding the publication of this paper.

\section{Supplementary Materials}

Approximations of the AFM nanoindentation regarding biological samples and biomaterials at the nanoscale. (Supplementary Materials)

\section{References}

[1] A. Stylianou, D. Yova, and K. Politopoulos, "Atomic force microscopy quantitative and qualitative nanoscale characterization of collagen thin films," in Proceedings of the 5th Conference on Emerging Technologies in NDT, CRC Press 2012, Chapter 65, pp. 415-420, 2012.

[2] D. P. Allison, N. P. Mortensen, C. J. Sullivan, and M. J. Doktycz, "Atomic force microscopy of biological samples," Wiley Interdisciplinary Reviews: Nanomedicine and Nanobiotechnology, vol. 2, no. 6, pp. 618-634, 2010.
[3] N. Gadegaard, "Atomic force microscopy in biology: technology and techniques," Biotechnic \& Histochemistry, vol. 81, no. 2-3, pp. 87-97, 2006.

[4] A. Stylianou and T. Stylianopoulos, "Atomic force microscopy probing of cancer cells and tumor microenvironment components," BioNanoScience, vol. 6, no. 1, pp. 33-46, 2016.

[5] A. Stylianou, D. Yova, and E. Alexandratou, "Nanotopography of collagen thin films in correlation with fibroblast response," Journal of Nanophotonics, vol. 7, no. 1, article 073590, 2013.

[6] G. Binnig, C. F. Quate, and C. Gerber, "Atomic force microscope," Physical Review Letters, vol. 56, no. 9, pp. 930-933, 1986.

[7] V. J. Morris, A. R. Kirby, and A. P. Gunning, Atomic Force Microscopy for Biologists, Imperial College Press, London, 2008.

[8] A. Stylianou, D. Yova, E. Alexandratou, and A. Petri, "Atomic force imaging microscopy investigation of the interaction of ultraviolet radiation with collagen thin ilms," in Nanoscale Imaging, Sensing, and Actuation for Biomedical Applications X, San Francisco, CA, USA, February 2013.

[9] A. Bergmann, D. Feigl, D. Kuhn et al., "A low-cost AFM setup with an interferometer for undergraduates and secondaryschool students," European Journal of Physics, vol. 34, no. 4, pp. 901-914, 2013.

[10] Y. Seo and W. Jhe, "Atomic force microscopy and spectroscopy," Reports on Progress in Physics, vol. 71, no. 1, article 016101, 2007.

[11] M. Minary-Jolandan and M. F. Yu, "Nanomechanical heterogeneity in the gap and overlap regions of type I collagen fibrils with implications for bone heterogeneity," Biomacromolecules, vol. 10, no. 9, pp. 2565-2570, 2009.

[12] F. M. Ohnesorge, "Towards atomic resolution non-contact dynamic force microscopy in a liquid," Surface and Interface Analysis, vol. 27, no. 5-6, pp. 379-385, 1999.

[13] W. C. Oliver and G. M. Pharr, "Measurement of hardness and elastic modulus by instrumented indentation: advances in understanding and refinements to methodology," Journal of Materials Research, vol. 19, no. 1, pp. 3-20, 2004.

[14] M. Stolz, R. Gottardi, R. Raiteri et al., "Early detection of aging cartilage and osteoarthritis in mice and patient samples using atomic force microscopy," Nature Nanotechnology, vol. 4, no. 3, pp. 186-192, 2009.

[15] M. Stolz, R. Raiteri, A. U. Daniels, M. R. Van Landingham, W. Baschong, and U. Aebi, "Dynamic elastic modulus of porcine articular cartilage determined at two different levels of tissue organization by indentation-type atomic force microscopy," Biophysical Journal, vol. 86, no. 5, pp. 32693283, 2004.

[16] C. Braunsmann, J. Seifert, J. Rheinlaender, and T. E. Schäffer, "High-speed force mapping on living cells with a small cantilever atomic force microscope," Review of Scientific Instruments, vol. 85, no. 7, article 073703, 2014.

[17] S. V. Kontomaris, A. Stylianou, D. Yova, and G. Balogiannis, "The effects of UV irradiation on collagen D-band revealed by atomic force microscopy," Scanning, vol. 37, no. 2, 111 pages, 2015.

[18] S. V. Kontomaris, A. Stylianou, D. Yova, and K. Politopoulos, "Mechanical properties of collagen fibrils on thin films by atomic force microscopy nanoindentation," in 2012 IEEE 
12th International Conference on Bioinformatics \& Bioengineering (BIBE), pp. 608-613, Larnaca, Cyprus, November 2012.

[19] S. V. Kontomaris, D. Yova, A. Stylianou, and K. Politopoulos, "The significance of the percentage differences of Young's modulus in the AFM nanoindentation procedure," Micro and Nanosystems, vol. 7, no. 2, pp. 86-97, 2015.

[20] A. Stylianou, S. V. Kontomaris, and D. Yova, "Assessing collagen nanoscale thin films heterogeneity by AFM multimode imaging and nanoindetation for nanobiomedical applications," Micro and Nanosystems, vol. 6, no. 2, pp. 95-102, 2014.

[21] D. Kirmizis and S. Logothetidis, "Atomic force microscopy probing in the measurement of cell mechanics," International Journal of Nanomedicine, vol. 5, no. 1, pp. 137-145, 2010.

[22] A. Stylianou and D. Yova, "Surface nanoscale imaging of collagen thin films by atomic force microscopy," Materials Science and Engineering C, vol. 33, no. 5, pp. 2947-2957, 2013.

[23] D. P. John and H. Georg, "Calibration of the torsional and lateral spring constants of cantilever sensors," Nanotechnology, vol. 25, no. 22, article 225701, 2014.

[24] S. Habelitz, M. Balooch, S. J. Marshall, G. Balooch, and G. W. Marshall Jr, "In situ atomic force microscopy of partially demineralized human dentin collagen fibrils," Journal of Structural Biology, vol. 138, no. 3, pp. 227-236, 2002.

[25] W. Melitz, J. Shen, A. C. Kummel, and S. Lee, "Kelvin probe force microscopy and its application," Surface Science Reports, vol. 66, no. 1, pp. 1-27, 2011.

[26] T. Yamaoka, H. Tsujikawa, S. Hasumura et al., "Vacuum magnetic force microscopy at high temperatures: observation of permanent magnets," Microscopy Today, vol. 22, no. 6, pp. 12-17, 2014.

[27] E. I. Altman, M. Z. Baykara, and U. D. Schwarz, "Noncontact atomic force microscopy: an emerging tool for fundamental catalysis research," Accounts of Chemical Research, vol. 48, no. 9, pp. 2640-2648, 2015.

[28] P. D. Garcia and R. Garcia, "Determination of the elastic moduli of a single cell cultured on a rigid support by force microscopy," Biophysical Journal, vol. 114, no. 12, pp. 2923-2932, 2018.

[29] S. E. Campbell, V. L. Ferguson, and D. C. Hurley, "Nanomechanical mapping of the osteochondral interface with contact resonance force microscopy and nanoindentation," Acta Biomaterialia, vol. 8, no. 12, pp. 4389-4396, 2012.

[30] M. O. Krisenko, A. Cartagena, A. Raman, and R. L. Geahlen, "Nanomechanical property maps of breast cancer cells as determined by multiharmonic atomic force microscopy reveal SYK-dependent changes in microtubule stability mediated by map1b," Biochemistry, vol. 54, no. 1, pp. 60-68, 2015.

[31] D. M. Harcombe, M. G. Ruppert, M. R. P. Ragazzon, and A. J. Fleming, "Higher-harmonic AFM imaging with a highbandwidth multifrequency Lyapunov filter," 2017 IEEE International Conference on Advanced Intelligent Mechatronics (AIM), 2017, pp. 725-730, Munich, Germany, July 2017.

[32] N. Shamsudhin, H. Rothuizen, B. J. Nelson, and A. Sebastian, "Multi-frequency atomic force microscopy: a system-theoretic approach," IFAC Proceedings Volumes, vol. 47, no. 3, pp. 7499-7504, 2014.

[33] A. Raman, S. Trigueros, A. Cartagena et al., "Mapping nanomechanical properties of live cells using multi-harmonic atomic force microscopy," Nature Nanotechnology, vol. 6, no. 12, pp. 809-814, 2011.

[34] S. Benaglia, V. G. Gisbert, A. P. Perrino, C. A. Amo, and R. Garcia, "Fast and high-resolution mapping of elastic properties of biomolecules and polymers with bimodal AFM," Nature Protocols, vol. 13, no. 12, pp. 2890-2907, 2018.

[35] Y. F. Dufrêne, D. Martínez-Martín, I. Medalsy, D. Alsteens, and D. J. Müller, "Multiparametric imaging of biological systems by force-distance curve-based AFM," Nature Methods, vol. 10, no. 9, pp. 847-854, 2013.

[36] R. García and R. Pérez, "Dynamic atomic force microscopy methods," Surface Science Reports, vol. 47, no. 6-8, pp. 197301, 2002.

[37] R. Garcia and R. Proksch, "Nanomechanical mapping of soft matter by bimodal force microscopy," European Polymer Journal, vol. 49, no. 8, pp. 1897-1906, 2013.

[38] G. Chawla and S. D. Solares, "Mapping of conservative and dissipative interactions in bimodal atomic force microscopy using open-loop and phase-locked-loop control of the higher eigenmode," Applied Physics Letters, vol. 99, no. 7, article 074103, 2011.

[39] Z. Al-Rekabi and S. Contera, "Multifrequency AFM reveals lipid membrane mechanical properties and the effect of cholesterol in modulating viscoelasticity," Proceedings of the National Academy of Sciences of the United States of America, vol. 115, no. 11, pp. 2658-2663, 2018.

[40] I. González-Domínguez, S. Gutiérrez-Granados, L. Cervera, F. Gòdia, and N. Domingo, "Identification of HIV-1-based virus-like particles by multifrequency atomic force microscopy," Biophysical Journal, vol. 111, no. 6, pp. 1173-1179, 2016.

[41] G. Lamour, C. K. Yip, H. Li, and J. Gsponer, "High intrinsic mechanical flexibility of mouse prion nanofibrils revealed by measurements of axial and radial Young's moduli," ACS Nano, vol. 8, no. 4, pp. 3851-3861, 2014.

[42] Z. Y. Zheng, R. Xu, S. L. Ye et al., "High harmonic exploring on different materials in dynamic atomic force microscopy," Science China Technological Sciences, vol. 61, no. 3, pp. 446452, 2018.

[43] J. Rother, H. Nöding, I. Mey, and A. Janshoff, "Atomic force microscopy-based microrheology reveals significant differences in the viscoelastic response between malign and benign cell lines," Open Biology, vol. 4, no. 5, 2014.

[44] U. Maver, T. Velnar, M. Gaberšček, O. Planinšek, and M. Finšgar, "Recent progressive use of atomic force microscopy in biomedical applications," TrAC Trends in Analytical Chemistry, vol. 80, pp. 96-111, 2016.

[45] M. Cascione, V. de Matteis, R. Rinaldi, and S. Leporatti, "Atomic force microscopy combined with optical microscopy for cells investigation," Microscopy Research and Technique, vol. 80, no. 1, pp. 109-123, 2017.

[46] S. Handschuh-Wang, T. Wang, and X. Zhou, "Recent advances in hybrid measurement methods based on atomic force microscopy and surface sensitive measurement techniques," RSC Advances, vol. 7, no. 75, pp. 47464-47499, 2017.

[47] L. Zhou, M. Cai, T. Tong, and H. Wang, "Progress in the correlative atomic force microscopy and optical microscopy," Sensors, vol. 17, no. 4, p. 938, 2017.

[48] J. W. Lichtman and J.-A. Conchello, "Fluorescence microscopy," Nature Methods, vol. 2, no. 12, pp. 910-919, 2005. 
[49] J.-A. Conchello and J. W. Lichtman, "Optical sectioning microscopy," Nature Methods, vol. 2, no. 12, pp. 920-931, 2005.

[50] J. Jonkman and C. M. Brown, "Any way you slice it-a comparison of confocal microscopy techniques," Journal of Biomolecular Techniques, vol. 26, no. 2, pp. 54-65, 2015.

[51] E. Alexandratou, D. Yova, and S. Loukas, "A confocal microscopy study of the very early cellular response to oxidative stress induced by zinc phthalocyanine sensitization," Free Radical Biology and Medicine, vol. 39, no. 9, pp. 1119-1127, 2005.

[52] J. R. Staunton, B. L. Doss, S. Lindsay, and R. Ros, "Correlating confocal microscopy and atomic force indentation reveals metastatic cancer cells stiffen during invasion into collagen I matrices," Scientific Reports, vol. 6, no. 1, article 19686, 2016.

[53] A. Stylianou, V. Gkretsi, and T. Stylianopoulos, "Transforming growth factor- $\beta$ modulates pancreatic cancer associated fibroblasts cell shape, stiffness and invasion," Biochimica et Biophysica Acta (BBA) - General Subjects, vol. 1862, no. 7, pp. 1537-1546, 2018.

[54] S. Cazaux, A. Sadoun, M. Biarnes-Pelicot et al., "Synchronizing atomic force microscopy force mode and fluorescence microscopy in real time for immune cell stimulation and activation studies," Ultramicroscopy, vol. 160, pp. 168-181, 2016.

[55] C. Canale, R. Oropesa-Nuñez, A. Diaspro, and S. Dante, "Amyloid and membrane complexity: the toxic interplay revealed by AFM," Seminars in Cell \& Developmental Biology, vol. 73, pp. 82-94, 2018.

[56] S. Wegmann, M. Miesbauer, K. F. Winklhofer, J. Tatzelt, and D. J. Muller, "Observing fibrillar assemblies on scrapieinfected cells," Pflügers Archiv - European Journal of Physiology, vol. 456, no. 1, pp. 83-93, 2008.

[57] M. S. Kuyukina, I. B. Ivshina, I. O. Korshunova, and E. V. Rubtsova, "Assessment of bacterial resistance to organic solvents using a combined confocal laser scanning and atomic force microscopy (CLSM/AFM)," Journal of Microbiological Methods, vol. 107, pp. 23-29, 2014.

[58] A. M. Streets, A. Li, T. Chen, and Y. Huang, "Imaging without fluorescence: nonlinear optical microscopy for quantitative cellular imaging," Analytical Chemistry, vol. 86, no. 17, pp. 8506-8513, 2014.

[59] A. Stylianou, K. Politopoulos, M. Kyriazi, and D. Yova, "Combined information from AFM imaging and SHG signal analysis of collagen thin films," Biomedical Signal Processing and Control, vol. 6, no. 3, pp. 307-313, 2011.

[60] R. Navab, D. Strumpf, To C et al., "Integrin $\alpha 11 \beta 1$ regulates cancer stromal stiffness and promotes tumorigenicity and metastasis in non-small cell lung cancer," Oncogene, vol. 35, no. 15, pp. 1899-1908, 2015.

[61] B. K. Robinson, E. Cortes, A. J. Rice, M. Sarper, and A. del Río Hernández, "Quantitative analysis of 3D extracellular matrix remodelling by pancreatic stellate cells," Biology Open, vol. 5, no. 6, pp. 875-882, 2016.

[62] N. R. Chevalier, E. Gazquez, L. Bidault et al., "Corrigendum: how tissue mechanical properties affect enteric neural crest cell migration," Scientific Reports, vol. 6, no. 1, article 34118, 2016.

[63] L. Liu, S. X. Zhang, W. Liao et al., "Mechanoresponsive stem cells to target cancer metastases through biophysical cues," Science Translational Medicine, vol. 9, no. 400, 2017.
[64] F. Braet, D. J. Taatjes, and E. Wisse, "Probing the unseen structure and function of liver cells through atomic force microscopy," Seminars in Cell \& Developmental Biology, vol. 73, pp. 13-30, 2018.

[65] M. Mahmoud, K. T. Daniela, M. Sascha, B. Mark, and H. Sonja, "3-D morphological characterization of the liver parenchyma by atomic force microscopy and by scanning electron microscopy," Microscopy Research and Technique, vol. 64, no. 1, pp. 1-9, 2004.

[66] T. V. Pavlova, I. A. Pavlo, N. B. Pilkevich, and M. A. Chaplygina, "Methods of scanning blood microscopy in oncology diagnosis of kidney pathology," Journal of Pharmacy Research, vol. 11, no. 12, pp. 1589-1593, 2017.

[67] R. Kaul-Ghanekar, S. Singh, H. Mamgain, A. Jalota-Badhwar, K. M. Paknikar, and S. Chattopadhyay, "Tumor suppressor protein SMAR1 modulates the roughness of cell surface: combined AFM and SEM study," BMC Cancer, vol. 9, no. 1, p. 350, 2009.

[68] S. Iyer, R. M. Gaikwad, V. Subba-Rao, C. D. Woodworth, and I. Sokolov, "Atomic force microscopy detects differences in the surface brush of normal and cancerous cells," Nature Nanotechnology, vol. 4, no. 6, pp. 389-393, 2009.

[69] L. I. Volakis, R. Li, A. WEt et al., "Loss of myoferlin redirects breast cancer cell motility towards collective migration," PLoS One, vol. 9, no. 2, article e86110, 2014.

[70] B. Alberts, A. Johnson, J. Lewis, P. Walter, M. Raff, and K. Roberts, Molecular Biology of the Cell, International Student Edition: Routledge, 4th edition, 2002.

[71] M. Radmacher, M. Fritz, J. P. Cleveland, D. A. Walters, and P. K. Hansma, "Imaging adhesion forces and elasticity of lysozyme adsorbed on mica with the atomic force microscope," Langmuir, vol. 10, no. 10, pp. 3809-3814, 1994.

[72] Y. Tatara, "Extensive theory of force- approach relations of elastic spheres in compression and in impact," Journal of Engineering Materials and Technology, vol. 111, no. 2, pp. 163-168, 1989.

[73] R. Afrin, M. T. Alam, and A. Ikai, "Pretransition and progressive softening of bovine carbonic anhydrase II as probed by single molecule atomic force microscopy," Protein Science, vol. 14, no. 6, pp. 1447-1457, 2005.

[74] A. Parra, E. Casero, E. Lorenzo, F. Pariente, and L. Vázquez, "Nanomechanical properties of globular proteins:lactate oxidase," Langmuir, vol. 23, no. 5, pp. 2747-2754, 2007.

[75] P. Fratzl, Collagen Structure and Mechanics, Springer, New York, NY, USA, 2008.

[76] D. J. S. Hulmes, "Collagen diversity, synthesis and assembly," in Collagen, pp. 15-47, Springer, Boston, MA, USA, 2008.

[77] S. Ricard-Blum, "The collagen family," Cold Spring Harbor Perspectives in Biology, vol. 3, no. 1, pp. 1-19, 2011.

[78] C. A. Grant, D. J. Brockwell, S. E. Radford, and N. H. Thomson, "Tuning the elastic modulus of hydrated collagen fibrils," Biophysical Journal, vol. 97, no. 11, pp. 2985-2992, 2009.

[79] M. G. Venugopal, J. A. M. Ramshaw, E. Braswell, D. Zhu, and B. Brodsky, "Electrostatic interactions in collagen-like triplehelical peptides," Biochemistry, vol. 33, no. 25, pp. 79487956, 1994.

[80] K. E. Kadler, C. Baldock, J. Bella, and R. P. Boot-Handford, "Collagens at a glance," Journal of Cell Science, vol. 120, no. 12, pp. 1955-1958, 2007.

[81] V. Hasirci, E. Vrana, P. Zorlutuna et al., "Nanobiomaterials: a review of the existing science and technology, and 
new approaches," Journal of Biomaterials Science, Polymer Edition, vol. 17, no. 11, pp. 1241-1268, 2006.

[82] S. Ricard-Blum, F. Ruggiero, and M. Van Der Rest, "The collagen superfamily," in Collagen, J. Brinckmann, H. Notbohm, and P. K. Müller, Eds., vol. 247 of Topics in Current Chemistry, pp. 35-84, Springer, Berlin, Heidelberg, 2005.

[83] L. Bozec, G. van der Heijden, and M. Horton, "Collagen fibrils: nanoscale ropes," Biophysical Journal, vol. 92, no. 1, pp. 70-75, 2007.

[84] C. A. Grant, M. A. Phillips, and N. H. Thomson, "Dynamic mechanical analysis of collagen fibrils at the nanoscale," Journal of the Mechanical Behavior of Biomedical Materials, vol. 5, no. 1, pp. 165-170, 2012.

[85] J. A. Petruska and A. J. Hodge, "A subunit model for the tropocollagen macromolecule," Proceedings of the National Academy of Sciences of the United States of America, vol. 51, no. 5, pp. 871-876, 1964.

[86] J. M. Wallace, B. G. Orr, J. C. Marini, and M. M. B. Holl, "Nanoscale morphology of type I collagen is altered in the Brtl mouse model of osteogenesis imperfecta," Journal of Structural Biology, vol. 173, no. 1, pp. 146-152, 2011.

[87] V. P. Ivanova and A. I. Krivchenko, "A current viewpoint on structure and evolution of collagens. I. Fibrillar collagens," Journal of Evolutionary Biochemistry and Physiology, vol. 48, no. 2, pp. 127-139, 2012.

[88] M. D. Shoulders and R. T. Raines, "Collagen structure and stability," Annual Review of Biochemistry, vol. 78, no. 1, pp. 929-958, 2009.

[89] M. K. Gordon and R. A. Hahn, "Collagens," Cell and Tissue Research, vol. 339, no. 1, pp. 247-257, 2010.

[90] A. Stylianou, "Atomic force microscopy for collagen-based nanobiomaterials," Journal of Nanomaterials, vol. 2017, Article ID 9234627, 14 pages, 2017.

[91] G. T. Tihan, I. RǍu, R. G. Zgârian, and M. V. Ghica, "Collagen-based biomaterials for ibuprofen delivery," Comptes Rendus Chimie, vol. 19, no. 3, pp. 390-394, 2016.

[92] B. D. Walters and J. P. Stegemann, "Strategies for directing the structure and function of three-dimensional collagen biomaterials across length scales," Acta Biomaterialia, vol. 10, no. 4, pp. 1488-1501, 2014.

[93] D. A. Cisneros, J. Friedrichs, A. Taubenberger, C. M. Franz, and D. J. Muller, "Creating ultrathin nanoscopic collagen matrices for biological and biotechnological applications," Small, vol. 3, no. 6, pp. 956-963, 2007.

[94] A. Stylianou, V. Gkretsi, and T. Stylianopoulos, "Atomic force microscopy nano-characterization of 3D collagen gels with tunable stiffness," MethodsX, vol. 5, pp. 503-513, 2018.

[95] N. E. Kurland, Z. Drira, and V. K. Yadavalli, "Measurement of nanomechanical properties of biomolecules using atomic force microscopy," Micron, vol. 43, no. 2-3, pp. 116-128, 2012.

[96] C. A. Grant, P. C. Twigg, and D. J. Tobin, "Static and dynamic nanomechanical properties of human skin tissue using atomic force microscopy: effect of scarring in the upper dermis," Acta Biomaterialia, vol. 8, no. 11, pp. 4123-4129, 2012.

[97] T. Ushiki, J. Hitomi, S. Ogura, T. Umemoto, and M. Shigeno, "Atomic force microscopy in histology and cytology," Archives of Histology and Cytology, vol. 59, no. 5, pp. 421431, 1996.

[98] B. E. Layton, A. M. Sastry, H. Wang et al., "Differences between collagen morphologies, properties and distribution in diabetic and normal biobreeding and Sprague-Dawley rat sciatic nerves," Journal of Biomechanics, vol. 37, no. 6, pp. 879-888, 2004.

[99] S. Choi, Y. Cheong, J. H. Shin et al., "Short-term nanostructural effects of high radiofrequency treatment on the skin tissues of rabbits," Lasers in Medical Science, vol. 27, no. 5, pp. 923-933, 2012.

[100] A. Sionkowska and T. Wess, "Mechanical properties of UV irradiated rat tail tendon (RTT) collagen," International Journal of Biological Macromolecules, vol. 34, no. 1-2, pp. 9-12, 2004.

[101] V. T. Natarajan, P. Ganju, A. Ramkumar, R. Grover, and R. S. Gokhale, "Multifaceted pathways protect human skin from UV radiation," Nature Chemical Biology, vol. 10, no. 7, pp. 542-551, 2014.

[102] E. D. Baron and A. K. Suggs, "Introduction to photobiology," Dermatologic Clinics, vol. 32, no. 3, pp. 255-266, 2014.

[103] D. Markovitsi, E. Sage, L. D. Frederick, and J. Davies, "Interaction of UV radiation with DNA," Photochemical \& Photobiological Sciences, vol. 12, no. 8, pp. 1256-1258, 2013.

[104] G. Fessel, J. Wernli, Y. Li, C. Gerber, and J. G. Snedeker, "Exogenous collagen cross-linking recovers tendon functional integrity in an experimental model of partial tear," Journal of Orthopaedic Research, vol. 30, no. 6, pp. 973-981, 2012.

[105] A. Gaspar, L. Moldovan, D. Constantin, A. M. Stanciuc, P. M. Sarbu Boeti, and I. C. Efrimescu, "Collagen-based scaffolds for skin tissue engineering," Journal of Medicine and Life, vol. 4, no. 2, pp. 172-177, 2011.

[106] H. Suh and J. C. Park, "Evaluation of calcification in porcine valves treated by ultraviolet ray and glutaraldehyde," Materials Science and Engineering C, vol. 13, no. 1-2, pp. 65-73, 2000.

[107] N. N. Fathima, R. Suresh, J. R. Rao, and B. U. Nair, "Effect of UV irradiation on the physicochemical properties of collagen stabilized using aldehydes," Journal of Applied Polymer Science, vol. 104, no. 6, pp. 3642-3648, 2007.

[108] N. N. Fathima, R. Suresh, J. R. Rao, B. U. Nair, and T. Ramasami, "Effect of UV irradiation on stabilized collagen: role of chromium (III)," Colloids and Surfaces B: Biointerfaces, vol. 62, no. 1, pp. 11-16, 2008.

[109] K. Jariashvili, B. Madhan, B. Brodsky, A. Kuchava, L. Namicheishvili, and N. Metreveli, "UV damage of collagen: insights from model collagen peptides," Biopolymers, vol. 97, no. 3, pp. 189-198, 2012.

[110] N. O. Metreveli, K. K. Jariashvili, L. O. Namicheishvili et al., "UV-vis and FT-IR spectra of ultraviolet irradiated collagen in the presence of antioxidant ascorbic acid," Ecotoxicology and Environmental Safety, vol. 73, no. 3, pp. 448-455, 2010.

[111] J. Skopinska-Wisniewska, A. Sionkowska, A. Kaminska, A. Kaznica, R. Jachimiak, and T. Drewa, "Surface characterization of collagen/elastin based biomaterials for tissue regeneration," Applied Surface Science, vol. 255, no. 19, pp. 8286-8292, 2009.

[112] A. Sionkowska, A. Płanecka, J. Kozłowska, and J. Skopińska-Wiśniewska, "Surface properties of UVirradiated poly (vinyl alcohol) films containing small amount of collagen," Applied Surface Science, vol. 255, no. 7, pp. 4135-4139, 2009.

[113] T. Stylianopoulos, J. D. Martin, V. P. Chauhan et al., "Causes, consequences, and remedies for growth-induced solid stress 
in murine and human tumors," Proceedings of the National Academy of Sciences of the United States of America, vol. 109, no. 38, pp. 15101-15108, 2012.

[114] A. Stylianou, D. Yova, and E. Alexandratou, "Investigation of the influence of UV irradiation on collagen thin films by AFM imaging," Materials Science and Engineering: C, vol. 45, pp. 455-468, 2014.

[115] K. Anselme, A. Ponche, and M. Bigerelle, "Relative influence of surface topography and surface chemistry on cell response to bone implant materials. Part 2: biological aspects," Proceedings of the Institution of Mechanical Engineers, Part $H$ : Journal of Engineering in Medicine, vol. 224, no. 12, pp. 1487-1507, 2010.

[116] C. Zink, H. Hall, D. M. Brunette, and N. D. Spencer, "Orthogonal nanometer-micrometer roughness gradients probe morphological influences on cell behavior," Biomaterials, vol. 33, no. 32, pp. 8055-8061, 2012.

[117] U. Covani, L. Giacomelli, A. Krajewski et al., "Biomaterials for orthopedics: a roughness analysis by atomic force microscopy," Journal of Biomedical Materials Research-Part A, vol. 82A, no. 3, pp. 723-730, 2007.

[118] Z. Cheng and S. H. Teoh, "Surface modification of ultra thin poly ( $\varepsilon$-caprolactone) films using acrylic acid and collagen," Biomaterials, vol. 25, no. 11, pp. 1991-2001, 2004.

[119] A. Sionkowska, J. Kozłowska, A. Płanecka, and J. SkopińskaWiśniewska, "Collagen fibrils in UV irradiated poly(vinyl pyrrolidone) films," Applied Surface Science, vol. 255, no. 5, pp. 2030-2039, 2008.

[120] K. Poole, K. Khairy, J. Friedrichs et al., "Molecular-scale topographic cues induce the orientation and directional movement of fibroblasts on two-dimensional collagen surfaces," Journal of Molecular Biology, vol. 349, no. 2, pp. 380-386, 2005.

[121] D. R. Stamov, A. Müller, Y. Wegrowski, S. Brezillon, and C. M. Franz, "Quantitative analysis of type I collagen fibril regulation by lumican and decorin using AFM," Journal of Structural Biology, vol. 183, no. 3, pp. 394-403, 2013.

[122] M. Achilli, J. Lagueux, and D. Mantovani, “On the effects of $\mathrm{UV}-\mathrm{C}$ and $\mathrm{pH}$ on the mechanical behavior, molecular conformation and cell viability of collagen-based scaffold for vascular tissue engineering," Macromolecular Bioscience, vol. 10, no. 3, pp. 307-316, 2010.

[123] A. B. Caruso and M. G. Dunn, "Changes in mechanical properties and cellularity during long-term culture of collagen fiber ACL reconstruction scaffolds," Journal of Biomedical Materials Research-Part A, vol. 73A, no. 4, pp. 388-397, 2005.

[124] S. Anastase-Ravion, M. P. Carreno, C. Blondin et al., "Synergistic effects of glucose and ultraviolet irradiation on the physical properties of collagen," Journal of Biomedical Materials Research, vol. 60, no. 3, pp. 384-391, 2002.

[125] D. P. Kuffler, "Photobiomodulation in promoting wound healing: a review," Regenerative Medicine, vol. 11, no. 1, pp. 107-122, 2016.

[126] K. Chawla, A. K. Lamba, S. Tandon, F. Faraz, and V. Gaba, "Effect of low-level laser therapy on wound healing after depigmentation procedure: a clinical study," Journal of Indian Society of Periodontology, vol. 20, no. 2, pp. 184-188, 2016.

[127] A. Stylianou and D. Yova, "Atomic force microscopy investigation of the interaction of low-level laser irradiation of collagen thin films in correlation with fibroblast response," Lasers in Medical Science, vol. 30, no. 9, pp. 2369-2379, 2015.

[128] M. K. Driscoll, X. Sun, C. Guven, J. T. Fourkas, and W. Losert, "Cellular contact guidance through dynamic sensing of nanotopography," ACS Nano, vol. 8, no. 4, pp. 3546-3555, 2014.

[129] F. Zhou, L. Yuan, H. Huang, and H. Chen, "Phenomenon of "contact guidance" on the surface with nano-micro-groovelike pattern and cell physiological effects," Chinese Science Bulletin, vol. 54, no. 18, pp. 3200-3205, 2009.

[130] S. Strasser, A. Zink, M. Janko, W. M. Heckl, and S. Thalhammer, "Structural investigations on native collagen type I fibrils using AFM," Biochemical and Biophysical Research Communications, vol. 354, no. 1, pp. 27-32, 2007.

[131] V. K. Yadavalli, D. V. Svintradze, and R. M. Pidaparti, "Nanoscale measurements of the assembly of collagen to fibrils," International Journal of Biological Macromolecules, vol. 46, no. 4, pp. 458-464, 2010.

[132] A. J. Heim, W. G. Matthews, and T. J. Koob, "Determination of the elastic modulus of native collagen fibrils via radial indentation," Applied Physics Letters, vol. 89, no. 18, article 181902, 2006.

[133] M. P. E. Wenger, L. Bozec, M. A. Horton, and P. Mesquidaz, "Mechanical properties of collagen fibrils," Biophysical Journal, vol. 93, no. 4, pp. 1255-1263, 2007.

[134] C. A. Grant, D. J. Brockwell, S. E. Radford, and N. H. Thomson, "Effects of hydration on the mechanical response of individual collagen fibrils," Applied Physics Letters, vol. 92, no. 23, article 233902, 2008.

[135] R. B. Svensson, T. Hassenkam, C. A. Grant, and S. P. Magnusson, "Tensile properties of human collagen fibrils and fascicles are insensitive to environmental salts," Biophysical Journal, vol. 99, no. 12, pp. 4020-4027, 2010.

[136] C. A. Grant, N. H. Thomson, M. D. Savage, H. W. Woon, and D. Greig, "Surface characterisation and biomechanical analysis of the sclera by atomic force microscopy," Journal of the Mechanical Behavior of Biomedical Materials, vol. 4, no. 4, pp. 535-540, 2011.

[137] C. A. Grant and P. C. Twigg, "Pseudostatic and dynamic nanomechanics of the tunica adventitia in elastic arteries using atomic force microscopy," ACS Nano, vol. 7, no. 1, pp. 456-464, 2013.

[138] P. G. Alexander, R. Gottardi, H. Lin, T. P. Lozito, and R. S. Tuan, "Three-dimensional osteogenic and chondrogenic systems to model osteochondral physiology and degenerative joint diseases," Experimental Biology and Medicine (Maywood, N.J.), vol. 239, no. 9, pp. 1080-1095, 2014.

[139] R. Gottardi, U. Hansen, R. Raiteri et al., "Supramolecular organization of collagen fibrils in healthy and osteoarthritic human knee and hip joint cartilage," PLoS One, vol. 11, no. 10, article e0163552, 2016.

[140] M. D. Buschmann, E. B. Hunziker, Y. J. Kim, and A. J. Grodzinsky, "Altered aggrecan synthesis correlates with cell and nucleus structure in statically compressed cartilage," Journal of Cell Science, vol. 109, pp. 499-508, 1996.

[141] F. Guilak, A. Ratcliffe, and V. C. Mow, "Chondrocyte deformation and local tissue strain in articular cartilage: a confocal microscopy study," Journal of Orthopaedic Research, vol. 13, no. 3, pp. 410-421, 1995.

[142] D. L. Garner and D. C. McGillivray, "Living articular cartilage is not smooth. The structure of mammalian and avian joint surfaces demonstrated in vivo by immersion incident light 
microscopy," Annals of the Rheumatic Diseases, vol. 30, no. 1, pp. 3-9, 1971.

[143] D. P. Speer and L. Dahners, "The collagenous architecture of articular cartilage. Correlation of scanning electron microscopy and polarized light microscopy observations," Clinical Orthopaedics and Related Research, no. 139, pp. 267-275, 1979.

[144] R. C. Appleyard, M. V. Swain, S. Khanna, and G. A. Murrell, "The accuracy and reliability of a novel handheld dynamic indentation probe for analysing articular cartilage," Physics in Medicine \& Biology, vol. 46, no. 2, pp. 541-550, 2001.

[145] R. M. Aspden, T. Larsson, R. Svensson, and D. Heinegård, "Computer-controlled mechanical testing machine for small samples of biological viscoelastic materials," Journal of Biomedical Engineering, vol. 13, no. 6, pp. 521-525, 1991.

[146] G. N. Duda, R. U. Kleemann, U. Bluecher, and A. Weiler, "A new device to detect early cartilage degeneration," The American Journal of Sports Medicine, vol. 32, no. 3, pp. 693-698, 2004.

[147] K. A. Athanasiou, C. F. Zhu, X. Wang, and C. M. Agrawal, "Effects of aging and dietary restriction on the structural integrity of rat articular cartilage," Annals of Biomedical Engineering, vol. 28, no. 2, pp. 143-149, 2000.

[148] R. V. Patel and J. J. Mao, "Microstructural and elastic properties of the extracellular matrices of the superficial zone of neonatal articular cartilage by atomic force microscopy," Frontiers in Bioscience, vol. 8, no. 1, pp. a18-a25, 2003.

[149] S. Tomkoria, R. V. Patel, and J. J. Mao, "Heterogeneous nanomechanical properties of superficial and zonal regions of articular cartilage of the rabbit proximal radius condyle by atomic force microscopy," Medical Engineering \& Physics, vol. 26, no. 10, pp. 815-822, 2004.

[150] M. Loparic, D. Wirz, A. U. Daniels et al., "Micro- and nanomechanical analysis of articular cartilage by indentation-type atomic force microscopy: validation with a gel-microfiber composite," Biophysical Journal, vol. 98, no. 11, pp. 27312740, 2010.

[151] F. Chen, X. Zhuang, L. Lin et al., "New horizons in tumor microenvironment biology: challenges and opportunities," BMC Medicine, vol. 13, no. 1, 2015.

[152] S. Kumar and V. M. Weaver, "Mechanics, malignancy, and metastasis: the force journey of a tumor cell," Cancer and Metastasis Reviews, vol. 28, no. 1-2, pp. 113-127, 2009.

[153] M. Lekka, "Discrimination between normal and cancerous cells using AFM," BioNano Science, vol. 6, no. 1, pp. 65-80, 2016.

[154] D. Hanahan and R. A. Weinberg, "Hallmarks of cancer: the next generation," Cell, vol. 144, no. 5, pp. 646-674, 2011.

[155] T. R. Cox, D. Bird, A. M. Baker et al., "LOX-mediated collagen crosslinking is responsible for fibrosis-enhanced metastasis," Cancer Research, vol. 73, no. 6, pp. 1721-1732, 2013.

[156] T. Stylianopoulos and R. K. Jain, "Combining two strategies to improve perfusion and drug delivery in solid tumors," Proceedings of the National Academy of Sciences of the United States of America, vol. 110, no. 46, pp. 18632-18637, 2013.

[157] V. Gkretsi, A. Stylianou, P. Papageorgis, C. Polydorou, and T. Stylianopoulos, "Remodeling components of the tumor microenvironment to enhance cancer therapy," Frontiers in Oncology, vol. 5, p. 214, 2015.
[158] J. G. Goetz, S. Minguet, I. Navarro-Lérida et al., "Biomechanical remodeling of the microenvironment by stromal caveolin-1 favors tumor invasion and metastasis," Cell, vol. 146, no. 1, pp. 148-163, 2011.

[159] A. Stylianou, V. Gkretsi, C. Patrickios, and T. Stylianopoulos, "Exploring the nano-surface of collagenous and other fibrotic tissues with AFM," in Fibrosis, L. Rittié, Ed., vol. 1627 of Methods in Molecular Biology, pp. 453-489, Humana Press, New York, NY, USA, 2017.

[160] A. Stylianou, M. Lekka, and T. Stylianopoulos, “AFM assessing of nanomechanical fingerprints for cancer early diagnosis and classification: from single cell to tissue level," Nanoscale, vol. 10, no. 45, pp. 20930-20945, 2018.

[161] M. Lekka, D. Gil, K. Pogoda et al., "Cancer cell detection in tissue sections using AFM," Archives of Biochemistry and Biophysics, vol. 518, no. 2, pp. 151-156, 2012.

[162] M. Plodinec, M. Loparic, C. A. Monnier et al., "The nanomechanical signature of breast cancer," Nature Nanotechnology, vol. 7, no. 11, pp. 757-765, 2012.

[163] S. Suresh, "Biomechanics and biophysics of cancer cells," Acta Biomaterialia, vol. 3, no. 4, pp. 413-438, 2007.

[164] I. Chasiotis, H. L. Fillmore, and G. T. Gillies, "Atomic force microscopy measurement of cytostructural elements involved in the nanodynamics of tumour cell invasion," Nanotechnology, vol. 14, no. 5, pp. 557-561, 2003.

[165] H. L. Fillmore, I. Chasiotis, S. W. Cho, and G. T. Gillies, "Atomic force microscopy observations of tumour cell invadopodia: novel cellular nanomorphologies on collagen substrates," Nanotechnology, vol. 14, no. 1, pp. 73-76, 2003.

[166] D. J. Müller and Y. F. Dufrêne, "Atomic force microscopy: a nanoscopic window on the cell surface," Trends in Cell Biology, vol. 21, no. 8, pp. 461-469, 2011.

[167] T. Jung, D. Castellana, P. Klingbeil et al., "CD44v6 dependence of premetastatic niche preparation by exosomes," Neoplasia, vol. 11, no. 10, pp. 1093-IN17, 2009.

[168] H. Peinado, M. Alečković, S. Lavotshkin et al., "Melanoma exosomes educate bone marrow progenitor cells toward a pro-metastatic phenotype through MET," Nature Medicine, vol. 18, no. 6, pp. 883-891, 2012.

[169] D. D. Taylor and C. Gercel-Taylor, "MicroRNA signatures of tumor-derived exosomes as diagnostic biomarkers of ovarian cancer," Gynecologic Oncology, vol. 110, no. 1, pp. 13-21, 2008.

[170] S. Sharma, K. Das, J. Woo, and J. K. Gimzewski, "Nanofilaments on glioblastoma exosomes revealed by peak force microscopy," Journal of the Royal Society Interface, vol. 11, no. $92,2014$.

[171] T. Ludwig, R. Kirmse, and K. Poole, "Challenges and approaches-probing tumor cell invasion by atomic force microscopy," in Modern Research and Educational Topics in Microscopy, Formatex, A. Méndez-Vilas and J. Díaz, Eds., pp. 11-22, 2007.

[172] S. Kusick, H. Bertram, H. Oberleithner, and T. Ludwig, "Nanoscale imaging and quantification of local proteolytic activity," Journal of Cellular Physiology, vol. 204, no. 3, pp. 767-774, 2005.

[173] T. Ludwig, "Local proteolytic activity in tumor cell invasion and metastasis," BioEssays, vol. 27, no. 11, pp. 1181-1191, 2005.

[174] A. V. Taubenberger, V. M. Quent, L. Thibaudeau, J. A. Clements, and D. W. Hutmacher, "Delineating breast 
cancer cell interactions with engineered bone microenvironments," Journal of Bone and Mineral Research, vol. 28, no. 6, pp. 1399-1411, 2013.

[175] S. Park, L. Bastatas, J. Matthews, and Y. J. Lee, "Mechanical responses of cancer cells on nanoscaffolds for adhesion size control," Macromolecular Bioscience, vol. 15, no. 6, pp. 851860, 2015.

[176] P. H. Puech, K. Poole, D. Knebel, and D. J. Muller, "A new technical approach to quantify cell-cell adhesion forces by AFM," Ultramicroscopy, vol. 106, no. 8-9, pp. 637-644, 2006.

[177] V. M. Laurent, A. Duperray, V. Sundar Rajan, and C. Verdier, "Atomic force microscopy reveals a role for endothelial cell ICAM-1 expression in bladder cancer cell adherence," PLoS One, vol. 9, no. 5, article e98034, 2014.

[178] S. C. Hoffmann, A. Cohnen, T. Ludwig, and C. Watzl, "2B4 engagement mediates rapid LFA-1 and actin-dependent NK cell adhesion to tumor cells as measured by single cell force spectroscopy," Journal of Immunology, vol. 186, no. 5, pp. 2757-2764, 2011.

[179] M. F. Coughlin, D. R. Bielenberg, G. Lenormand et al., "Cytoskeletal stiffness, friction, and fluidity of cancer cell lines with different metastatic potential," Clinical and Experimental Metastasis, vol. 30, no. 3, pp. 237-250, 2013.

[180] M. Plodinec and R. Y. H. Lim, "Nanomechanical characterization of living mammary tissues by atomic force microscopy," in Mammary Stem Cells, M. Vivanco, Ed., vol. 1293 of Methods in Molecular Biology, pp. 231-246, Humana Press, New York, NY, USA, 2015.

[181] A. Fuhrmann, J. R. Staunton, V. Nandakumar, N. Banyai, P. C. W. Davies, and R. Ros, "AFM stiffness nanotomography of normal, metaplastic and dysplastic human esophageal cells," Physical Biology, vol. 8, no. 1, article 015007, 2011.

[182] K. Hayashi and M. Iwata, "Stiffness of cancer cells measured with an AFM indentation method," Journal of the Mechanical Behavior of Biomedical Materials, vol. 49, pp. 105-111, 2015.

[183] W. H. Goldmann and R. M. Ezzell, "Viscoelasticity in wild-type and vinculin-deficient (5.51) mouse F9 embryonic carcinoma cells examined by atomic force microscopy and rheology," Experimental Cell Research, vol. 226, no. 1, pp. 234-237, 1996.

[184] W. H. Goldmann, R. Galneder, M. Ludwig et al., "Differences in elasticity of vinculin-deficient F9 cells measured by magnetometry and atomic force microscopy," Experimental Cell Research, vol. 239, no. 2, pp. 235-242, 1998.

[185] M. Lekka, P. Laidler, D. Gil, J. Lekki, Z. Stachura, and A. Z. Hrynkiewicz, "Elasticity of normal and cancerous human bladder cells studied by scanning force microscopy," European Biophysics Journal, vol. 28, no. 4, pp. 312-316, 1999.

[186] M. Lekka, J. Lekki, M. Marszałek et al., "Local elastic properties of cells studied by SFM," Applied Surface Science, vol. 141, no. 3-4, pp. 345-349, 1999.

[187] Q. S. Li, G. Y. H. Lee, C. N. Ong, and C. T. Lim, “AFM indentation study of breast cancer cells," Biochemical and Biophysical Research Communications, vol. 374, no. 4, pp. 609-613, 2008.

[188] E. C. Faria, N. Ma, E. Gazi et al., "Measurement of elastic properties of prostate cancer cells using AFM," Analyst, vol. 133, no. 11, pp. 1498-1500, 2008.
[189] Z. L. Zhou, A. H. W. Ngan, B. Tang, and A. X. Wang, "Reliable measurement of elastic modulus of cells by nanoindentation in an atomic force microscope," Journal of the Mechanical Behavior of Biomedical Materials, vol. 8, pp. 134-142, 2012.

[190] S. E. Cross, Y.-S. Jin, J. Rao, and J. K. Gimzewski, "Nanomechanical analysis of cells from cancer patients," Nature Nanotechnology, vol. 2, no. 12, pp. 780-783, 2007.

[191] K. Pogoda, J. Jaczewska, J. Wiltowska-Zuber et al., "Depthsensing analysis of cytoskeleton organization based on AFM data," European Biophysics Journal, vol. 41, no. 1, pp. 79-87, 2012.

[192] S. Park and Y. J. Lee, "AFM-based dual nano-mechanical phenotypes for cancer metastasis," Journal of Biological Physics, vol. 40, no. 4, pp. 413-419, 2014.

[193] K. Levental, H. Yu, L. Kass et al., "Matrix crosslinking forces tumor progression by enhancing integrin signaling," Cell, vol. 139, no. 5, pp. 891-906, 2009.

[194] M. J. Paszek, N. Zahir, K. R. Johnson et al., "Tensional homeostasis and the malignant phenotype," Cancer Cell, vol. 8, no. 3, pp. 241-254, 2005.

[195] T. Stylianopoulos, L. L. Munn, and R. K. Jain, "Reengineering the physical microenvironment of tumors to improve drug delivery and efficacy: from mathematical modeling to bench to bedside," Trends in Cancer, vol. 4, no. 4, pp. 292-319, 2018.

[196] M. Lekka, "Atomic force microscopy: a tip for diagnosing cancer," Nature Nanotechnology, vol. 7, no. 11, pp. 691-692, 2012.

[197] A. Ansardamavandi, M. Tafazzoli-Shadpour, R. Omidvar, and I. Jahanzad, "Quantification of effects of cancer on elastic properties of breast tissue by atomic force microscopy," Journal of the Mechanical Behavior of Biomedical Materials, vol. 60, pp. 234-242, 2016.

[198] J. I. Lopez, I. Kang, W. K. You, D. M. McDonald, and V. M. Weaver, "In situ force mapping of mammary gland transformation," Integrative Biology, vol. 3, no. 9, pp. 910-921, 2011.

[199] M. Tian, Y. Li, W. Liu et al., "The nanomechanical signature of liver cancer tissues and its molecular origin," Nanoscale, vol. 7, no. 30, pp. 12998-13010, 2015.

[200] G. Ciasca, T. E. Sassun, E. Minelli et al., "Nano-mechanical signature of brain tumours," Nanoscale, vol. 8, no. 47, pp. 19629-19643, 2016.

[201] Y. Cui, X. Zhang, K. You et al., "Nanomechanical characteristics of cervical cancer and cervical intraepithelial neoplasia revealed by atomic force microscopy," Medical Science Monitor: International Medical Journal of Experimental and Clinical Research, vol. 23, pp. 4205-4213, 2017.

[202] E. Minelli, G. Ciasca, T. E. Sassun et al., "A fully-automated neural network analysis of AFM force-distance curves for cancer tissue diagnosis," Applied Physics Letters, vol. 111, no. 14, article 143701, 2017.

[203] J. Adamcik and R. Mezzenga, "Study of amyloid fibrils via atomic force microscopy," Current Opinion in Colloid \& Interface Science, vol. 17, no. 6, pp. 369-376, 2012.

[204] S. J. Roeters, A. Iyer, G. Pletikapiä, V. Kogan, V. Subramaniam, and S. Woutersen, "Evidence for intramolecular antiparallel beta-sheet structure in alphasynuclein fibrils from a combination of two-dimensional infrared spectroscopy and atomic force microscopy," Scientific Reports, vol. 7, no. 1, 2017. 
[205] H. Lei, X. Zhang, J. Hu, and Y. Zhang, "Self-assembly of amyloid-like peptides at interfaces investigated by atomic force microscopy," Science of Advanced Materials, vol. 9, no. 1, pp. 65-76, 2017.

[206] T. Watanabe-Nakayama and K. Ono, "High-speed atomic force microscopy of individual amyloidogenic protein assemblies," in Nanoscale Imaging, Y. Lyubchenko, Ed., vol. 1814 of Methods in Molecular Biology, pp. 201-212, Humana Press, New York, NY, USA, 2018.

[207] S. W. Han, T. H. Lee, M. S. Kang, H. J. Kim, and H. K. Shin, "Probing amyloid $\beta$ and the antibody interaction using atomic force microscopy," Journal of Nanoscience and Nanotechnology, vol. 18, no. 2, pp. 1410-1413, 2018.

[208] E. Drolle, F. Hane, B. Lee, and Z. Leonenko, "Atomic force microscopy to study molecular mechanisms of amyloid fibril formation and toxicity in Alzheimer's disease," Drug Metabolism Reviews, vol. 46, no. 2, pp. 207-223, 2014.

[209] A. P. Shivji, M. C. Davies, C. J. Roberts, S. J. B. Tendler, and M. J. Wilkinson, "Molecular surface morphology studies of $\beta$-amyloid self-assembly: effect of $\mathrm{pH}$ on fibril formation," Protein and Peptide Letters, vol. 3, no. 6, pp. 407-414, 1996.

[210] W. B. Stine Jr, S. W. Snyder, U. S. Ladror et al., "The nanometer-scale structure of amyloid- $\beta$ visualized by atomic force microscopy," Protein Journal, vol. 15, no. 2, pp. 193203, 1996.

[211] G. S. Shekhawat, M. P. Lambert, S. Sharma et al., "Soluble state high resolution atomic force microscopy study of Alzheimer's $\beta$-amyloid oligomers," Applied Physics Letters, vol. 95, no. 18, article 183701, 2009.

[212] G. M. Mustata, G. S. Shekhawat, M. P. Lambert et al., "Insights into the mechanism of Alzheimer's $\beta$-amyloid aggregation as a function of concentration by using atomic force microscopy," Applied Physics Letters, vol. 100, no. 13, article 133704, 2012.

[213] L. Connelly, H. Jang, F. Teran Arce et al., “Atomic force microscopy and MD simulations reveal pore-like structures of all-d-enantiomer of Alzheimer's $\beta$-amyloid peptide: relevance to the ion channel mechanism of AD pathology," Journal of Physical Chemistry B, vol. 116, no. 5, pp. 17281735, 2012.

[214] E. Drolle, K. Hammond, F.T Hane, B. Lee, and Z. Leonenko, "Atomic Force Microscopy and Kelvin Probe Force Microscopy to Study Molecular Mechanism of Alzheimer's Disease," in International Conference on Scanning Probe Microscopy on Soft and Polymeric Materials, Ontario, Canada, September 2014.

[215] S. Song, X. Ma, Y. Zhou, M. Xu, S. Shuang, and C. Dong, "Studies on the interaction between vanillin and $\beta$-amyloid protein via fluorescence spectroscopy and atomic force microscopy," Chemical Research in Chinese Universities, vol. 32, no. 2, pp. 172-177, 2016.

[216] S. W. Han, H. K. Shin, and T. Adachi, "Nanolithography of amyloid precursor protein cleavage with $\beta$-secretase by atomic force microscopy," Journal of Biomedical Nanotechnology, vol. 12, no. 3, pp. 546-553, 2016.

[217] N. Li, H. Jang, M. Yuan et al., "Graphite-templated amyloid nanostructures formed by a potential pentapeptide inhibitor for Alzheimer's disease: a combined study of real-time atomic force microscopy and molecular dynamics simulations," Langmuir, vol. 33, no. 27, pp. 6647-6656, 2017.
[218] P. J. de Pablo, "Atomic force microscopy of virus shells," Seminars in Cell \& Developmental Biology, vol. 73, pp. 199-208, 2018.

[219] F. Moreno-Madrid, N. Martín-González, A. Llauró et al., "Atomic force microscopy of virus shells," Biochemical Society Transactions, vol. 45, no. 2, pp. 499-511, 2017.

[220] M. G. Mateu, "Mechanical properties of viruses analyzed by atomic force microscopy: a virological perspective," Virus Research, vol. 168, no. 1-2, pp. 1-22, 2012.

[221] A. M. Baró, R. Miranda, J. Alamán et al., "Determination of surface topography of biological specimens at high resolution by scanning tunnelling microscopy," Nature, vol. 315, no. 6016, pp. 253-254, 1985.

[222] Y. G. Kuznetsov, A. J. Malin, R. W. Lucas, M. Plomp, and A. McPherson, "Imaging of viruses by atomic force microscopy," Journal of General Virology, vol. 82, no. 9, pp. 20252034, 2001.

[223] Y. G. Kuznetsov and A. McPherson, "Atomic force microscopy in imaging of viruses and virus-infected cells," Microbiology and Molecular Biology Reviews, vol. 75, no. 2, pp. 268-285, 2011.

[224] A. J. Malkin, M. Plomp, and A. McPherson, "Unraveling the architecture of viruses by high-resolution atomic force microscopy," in DNA Viruses, P. M. Lieberman, Ed., vol. 292 of Methods in Molecular Biology, , pp. 85-108, Humana Press, 2005.

[225] A. McPherson and Y. G. Kuznetsov, "Atomic force microscopy investigation of viruses," in Atomic Force Microscopy in Biomedical Research, P. Braga and D. Ricci, Eds., vol. 736 of Methods in Molecular Biology (Methods and Protocols), , pp. 171-195, Humana Press, 2011.

[226] M. Gladnikoff and I. Rousso, "Directly monitoring individual retrovirus budding events using atomic force microscopy," Biophysical Journal, vol. 94, no. 1, pp. 320-326, 2008.

[227] M. Gladnikoff, E. Shimoni, N. S. Gov, and I. Rousso, "Retroviral assembly and budding occur through an actin-driven mechanism," Biophysical Journal, vol. 97, no. 9, pp. 24192428, 2009.

[228] W. Häberle, J. K. H. Hörber, F. Ohnesorge, D. P. E. Smith, and G. Binnig, "In situ investigations of single living cells infected by viruses," Ultramicroscopy, vol. 42-44, pp. 11611167, 1992.

[229] M. Moloney, L. McDonnell, and H. O'Shea, “Atomic force microscopy analysis of enveloped and non-enveloped viral entry into, and egress from, cultured cells," Ultramicroscopy, vol. 100, no. 3-4, pp. 163-169, 2004.

[230] R. D. Hartschuh, S. P. Wargacki, H. Xiong et al., "How rigid are viruses," Physical Review E, vol. 78 , no. 2, article 021907, 2008.

[231] R. Ramalho, S. Rankovic, J. Zhou, C. Aiken, and I. Rousso, "Analysis of the mechanical properties of wild type and hyperstable mutants of the HIV-1 capsid," Retrovirology, vol. 13, no. 1, 2016.

[232] B. K. Ganser, S. Li, V. Y. Klishko, J. T. Finch, and W. I. Sundquist, "Assembly and analysis of conical models for the HIV-1 core," Science, vol. 283, no. 5398, pp. 80-83, 1999.

[233] W. I. Sundquist and H. G. Kräusslich, "HIV-1 assembly, budding, and maturation," Cold Spring Harbor Perspectives in Medicine, vol. 2, no. 7, 2012.

[234] G. Zhao, J. R. Perilla, E. L. Yufenyuy et al., "Mature HIV-1 capsid structure by cryo-electron microscopy and all-atom 
molecular dynamics," Nature, vol. 497, no. 7451, pp. 643646, 2013.

[235] H. B. Pang, L. Hevroni, N. Kol et al., "Virion stiffness regulates immature HIV-1 entry," Retrovirology, vol. 10, no. 1, p. 4, 2013.

[236] N. Kol, Y. Shi, M. Tsvitov et al., "A stiffness switch in human immunodeficiency virus,” Biophysical Journal, vol. 92, no. 5, pp. 1777-1783, 2007.

[237] V. Gkretsi, A. Stylianou, and T. Stylianopoulos, "Vasodilatorstimulated phosphoprotein (VASP) depletion from breast cancer MDA-MB-231 cells inhibits tumor spheroid invasion through downregulation of Migfilin, $\beta$-catenin and urokinase-plasminogen activator (uPA)," Experimental Cell Research, vol. 352, no. 2, pp. 281-292, 2017. 

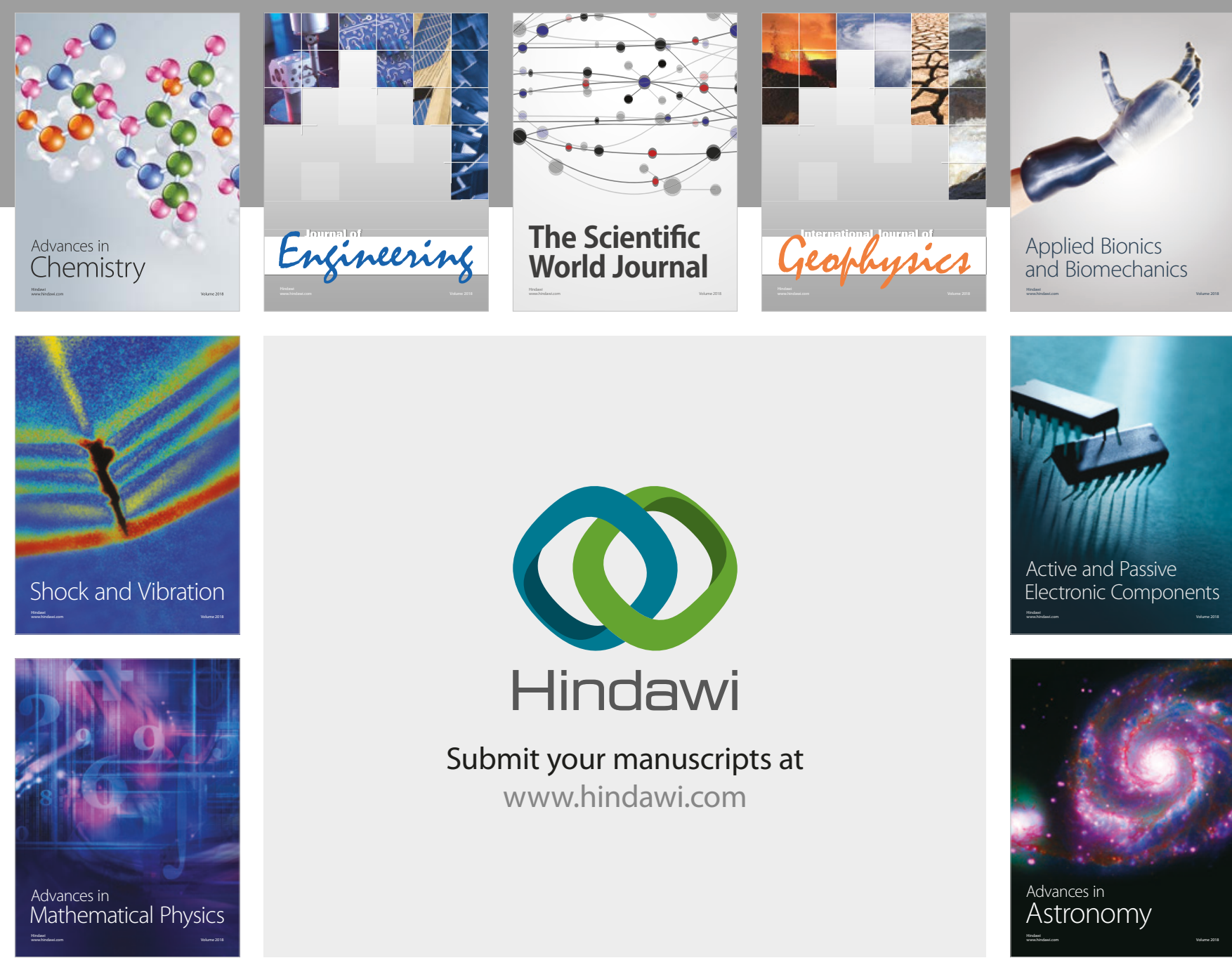

Submit your manuscripts at

www.hindawi.com

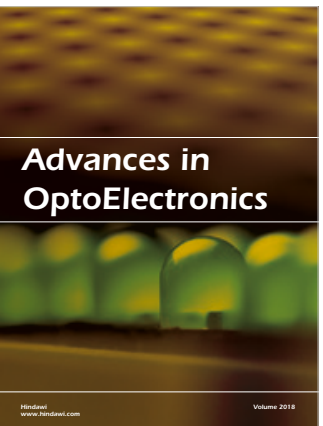

\section{Rotcting Machinery}
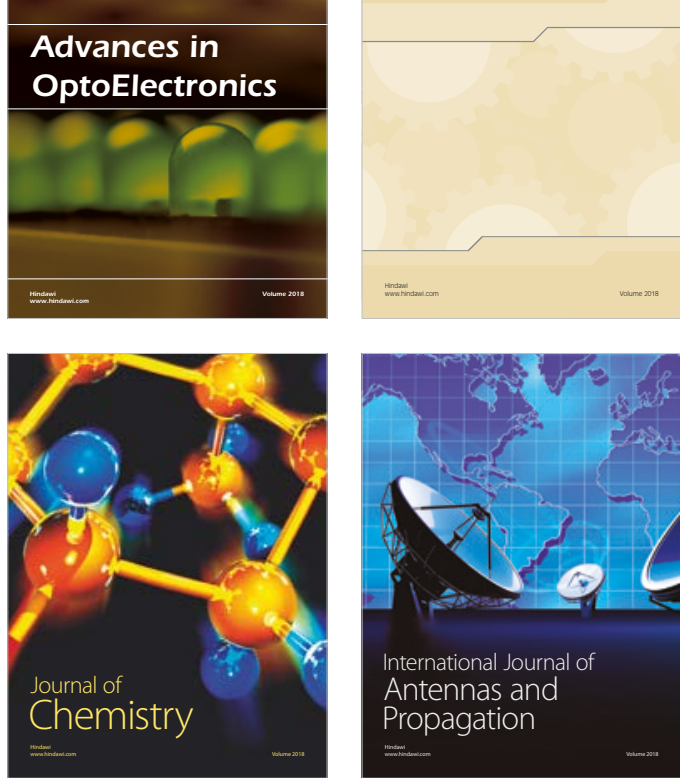

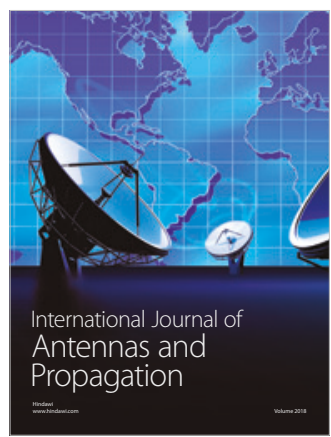

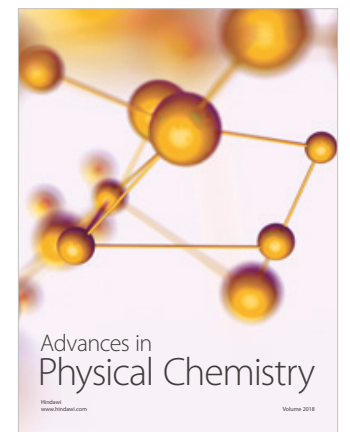

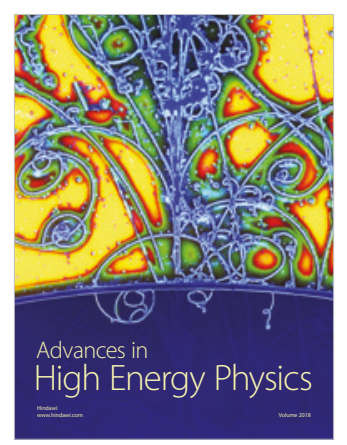

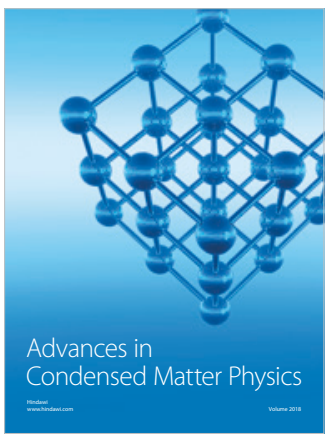

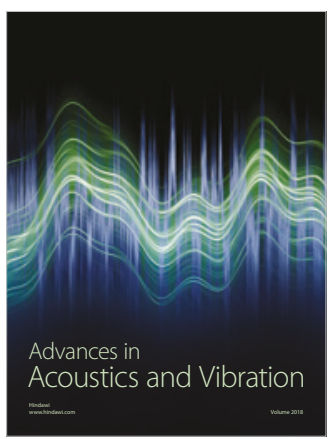

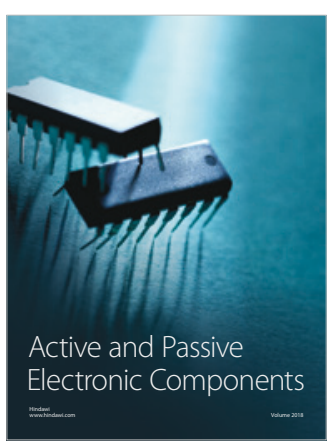
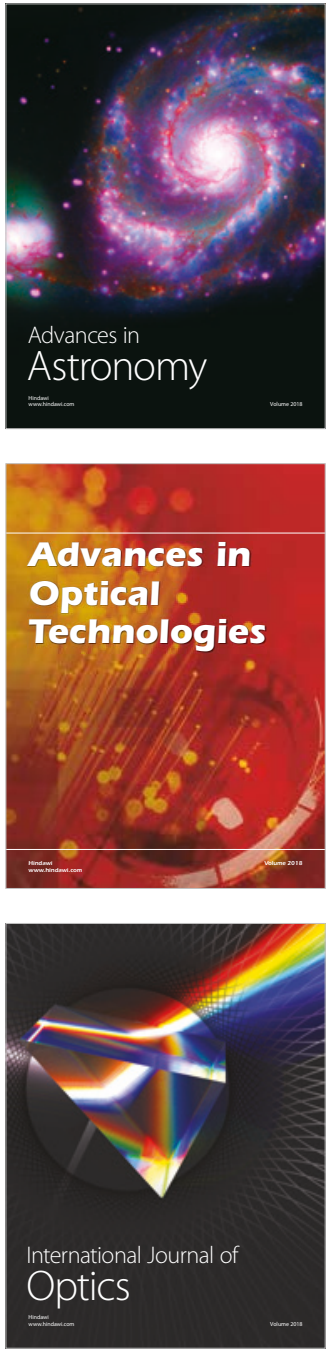